\title{
Transition to chaos in the cylinder wake through the Mode $\mathrm{C}$ \\ flow
}

\author{
HONGYI JIANG (蒋弘毅) ${ }^{1}$ and LIANG CHENG (程亮 $)^{1,2 \dagger}$ \\ ${ }^{1}$ School of Engineering, The University of Western Australia, 35 Stirling Highway, Crawley, WA \\ 6009, Australia
}

${ }^{2}$ State Key Laboratory of Coastal and Offshore Engineering, Dalian University of Technology, Dalian, 116024, China

\begin{abstract}
The evolution of Mode $\mathrm{C}$ wake characteristics with the Reynolds number $(R e)$ for $R e$ up to 400 is investigated numerically. The Mode C wake instability is generated by placing a small wire in the near wake of a main circular cylinder. This setup ensures that the wake is unstable to Mode C only (without potential mode interactions), as demonstrated by Floquet stability analysis. Based on three-dimensional direct numerical simulations, three evolution regimes are identified for the fully developed Mode C flow. In the uniform periodic regime $(R e=166.4-210)$, the Mode $C$ structure is uniformly distributed along the spanwise direction. The flow structure is $2 T$-periodic ( $T$ being the vortex shedding period) but retains a spatiotemporal symmetry every $1 T$. In the non-uniform periodic regime $(R e=220-230)$, the slightly non-uniform Mode $\mathrm{C}$ structure remains 2T-periodic at $R e=220$ but undergoes a period quadrupling to $4 T$-periodic at $R e=230$ before transitioning to chaos. In the chaotic regime $(R e \geq 240)$, the flow loses periodicity and becomes increasingly chaotic with increasing $R e$. The progressive wake transition to chaos is found to originate from the instability in the braid shear layer region, through the uneven growth in strength and the consequent non-uniformity of the Mode $\mathrm{C}$ streamwise vortices. The wake transitions to chaos through the routes of Mode C and Mode B (for an isolated circular/square cylinder) are compared.
\end{abstract}

\footnotetext{
†Email address for correspondence: liang.cheng@uwa.edu.au
} 


\section{Introduction}

Steady incoming flow past a nominally two-dimensional (2D) bluff body has been a classical topic in fluid mechanics. In addition to its extensive practical applications, the phenomenon of wake transition to turbulence/chaos is of great fundamental importance. The scenario of steady incoming flow past a long smooth cylinder is governed by a single dimensionless parameter, the Reynolds number $\operatorname{Re}(=$ $U D / v)$, which is defined based on the incoming flow velocity $(U)$, the length scale of the cylinder perpendicular to the incoming flow $(D)$, and the kinematic viscosity of the fluid $(v)$.

For the canonical case of flow past a circular cylinder, with increasing $R e$ three-dimensional (3D) wake transition occurs in the following sequence (see, e.g., Williamson, 1996; Henderson, 1997; Barkley et al., 2000):

(i) At $R e \sim 190$, the flow transitions from 2D to 3D through the Mode A instability that originates in the primary vortex cores (Williamson, 1996; Leweke and Williamson, 1998; Thompson et al., 2001). The Mode A instability is subcritical in nature and contains a hysteresis loop of an $R e$ interval of 5 (Henderson and Barkley, 1996; Henderson, 1997; Akbar et al., 2011). The relatively large-scale Mode A structure has a spanwise wavelength of $\sim 4 D$ at its onset. The spanwise wavelength decreases slightly with increase in $\operatorname{Re}$ (Williamson, 1996; Barkley and Henderson, 1996). Each spanwise wavelength consists of two streamwise vortices with opposite signs that form a streamwise vortex pair/loop. The neighbouring streamwise vortices in the streamwise direction have an out-of-phase sequence. The ordered Mode A structure is unstable over time and will evolve spontaneously into a more stable pattern with vortex dislocations. The Mode A flow with vortex dislocations is observed for the fully developed flows of $R e \sim 190$ to 230 .

(ii) At $R e \sim 230$ to 260 , a gradual wake transition from Mode A with vortex dislocations to the finer-scale Mode B flow takes place. The Mode B 
instability originates in the braid shear layer region (Williamson, 1996; Leweke and Williamson, 1998; Thompson et al., 2001) and is supercritical (Henderson, 1997). The Mode B structure has a spanwise wavelength of $0.8 D$ (for a streamwise vortex pair) and an in-phase sequence between the neighbouring streamwise vortices in the streamwise direction.

(iii) At $R e \sim 260$, the pattern of Mode A with vortex dislocations disappears. A critical condition is observed at $R e \sim 260$, where the Mode B flow structures are particularly ordered. For $R e \gtrsim 260$, the Mode B structures become increasingly disordered with increasing $R e$ and the flow becomes increasingly chaotic. The Mode B pattern remains in the wake up to at least $R e=10000$ (Williamson, 1996).

For other bluff-body geometries, wake instability modes other than Modes A and B may develop. One of the commonly observed wake instability modes is Mode C. The most striking feature of Mode $\mathrm{C}$ is that it is $2 T$-periodic ( $T$ being the vortex shedding period) (see e.g. Sheard et al., 2005a). The Mode C instability is often observed when the $Z_{2}$ spatiotemporal symmetry of the wake (spatial reflection of the flow about the wake centreline after every $T / 2$ time evolution) is broken by a perturbation imposed on the bluff body (Blackburn and Sheard, 2010). The perturbation can normally be generated by a geometric configuration or a movement that is asymmetric about the wake centreline, e.g. a circular cylinder with a trip-wire in the near-wake but offset from the wake centreline (Zhang et al., 1995; Yildirim et al., 2013a,b), a circular ring (Sheard et al., 2003, 2004, 2005b), two circular cylinders in staggered arrangements (Carmo et al., 2008), a rotated square cylinder (Sheard et al., 2009), a rotating circular cylinder (Rao et al., 2013), a circular cylinder in proximity to a slip wall (Jiang et al., 2017a), a rotated elliptical cylinder (Rao et al., 2017), a circular cylinder subjected to planar shear flow (Park and Yang, 2018), a rotated triangular cylinder ( $\mathrm{Ng}$ et al., 2018), etc.

The Mode $\mathrm{C}$ instability reported in most of the studies was identified using linear stability analysis (e.g. Sheard et al., 2003; Carmo et al., 2008; Sheard et al., 2009; Rao 
et al., 2013; Rao et al., 2017; Park and Yang, 2018; Ng et al., 2018). On the other hand, the non-linear evolution of the Mode $\mathrm{C}$ flow structure with $R e$ (including the wake transition to chaos through the Mode $\mathrm{C}$ flow) has been rarely explored. The Mode $\mathrm{C}$ wake instability was first observed both experimentally (with $D_{w} / D=0.006$, where $D_{w}$ is the diameter of the wire) and numerically (with $D_{w} / D=0.05$ ) by Zhang et al. (1995) through placing a trip-wire at $(x / D, y / D)=(0.75,0.75)$ in the near wake of a main circular cylinder centred at $(x / D, y / D)=(0,0)$. In their experiments, the Mode $\mathrm{C}$ structure was observed over a range of $R e$ from 170 to 270 with a spanwise wavelength of approximately $2 D$. The Mode $\mathrm{C}$ flow was periodic for $170<R e<200$ and quasi-periodic for $200<R e<270$. Based on their numerical study, the spanwise wavelength of Mode $\mathrm{C}$ was $1.8 D$ at $R e=210$. A more recent experimental study by Yildirim et al. (2013a), where a trip-wire of $D_{w} / D=0.01$ was placed at the same near-wake location to that in Zhang et al. (1995), observed the Mode C structure over a range of $R e$ from its onset point of $R e=165-180$ to the largest $R e$ of 300 considered in their study. In contrast to Zhang et al. (1995), Yildirim et al. (2013a) found that the Mode $\mathrm{C}$ structure became increasingly disordered with increasing $R e$, whereas no drastic change of the vortex structure was detected. The spanwise wavelength of Mode $\mathrm{C}$ was approximately $2 D$ according to Yildirim et al. (2013a) and was found to scatter between $1.8 D$ and $2.4 D$ in Yildirim et al. (2013b) after further analysis. Yildirim et al. (2013a) also suggested that the feedback of streamwise vortices between two consecutive primary vortices on the wire side is the reason for the self-sustaining of the Mode $\mathrm{C}$ structure at the same spanwise locations.

In light of the earlier works, the primary aim of this study is to investigate in detail the non-linear evolution of the Mode $\mathrm{C}$ flow structure with $R e$, with a particular focus on how the wake progressively transitions to chaos through the Mode C flow. In the present study, the Mode $\mathrm{C}$ flow is generated based on the geometric setup of Zhang et al. (1995) and Yildirim et al. (2013a,b), namely a circular cylinder with a near-wake wire disturbance. As demonstrated in section 3.1.2 through Floquet stability analysis, this setup ensures that the wake is unstable to Mode $\mathrm{C}$ only, without 
potential interactions with other modes. In the absence of Mode B that is responsible for the wake transition to chaos of an isolated circular/square cylinder (e.g. Williamson, 1996; Jiang et al., 2018), the wake transition to chaos through the route of the Mode C flow is investigated and compared with that of the Mode B flow.

\section{Numerical model}

\subsection{Numerical method}

OpenFOAM (www.openfoam.org) has been used in this study in solving the continuity and incompressible Navier-Stokes equations:

$\frac{\partial u_{i}}{\partial x_{i}}=0$

$\frac{\partial u_{i}}{\partial t}+u_{j} \frac{\partial u_{i}}{\partial x_{j}}=-\frac{1}{\rho} \frac{\partial p}{\partial x_{i}}+v \frac{\partial^{2} u_{i}}{\partial x_{j} \partial x_{j}}$

where $\left(x_{1}, x_{2}, x_{3}\right)=(x, y, z)$ are Cartesian coordinates, $u_{i}$ is the velocity component in the direction $x_{i}, t$ is time, $\rho$ is fluid density, and $p$ is pressure. The Finite Volume Method (FVM) and the Pressure Implicit with Splitting of Operators (PISO) algorithm (Issa, 1986) are used to solve the equations. The convection, diffusion and time derivative terms are discretized, respectively, using a fourth-order cubic scheme, a second-order linear scheme, and a blended scheme consisting of the second-order Crank-Nicolson scheme and a first-order Euler implicit scheme. The same numerical approach has been used in Jiang et al. $(2016,2018)$ and Jiang and Cheng (2018) for the simulations of wake transition of a circular and a square cylinder.

\subsection{Computational domain and boundary conditions}

A hexahedral computational domain of $50 D \times 40 D \times 12 D$, as shown in Fig. 1(a), is adopted for the present direct numerical simulations (DNS). The centre of the main circular cylinder is located at $(x / D, y / D)=(0,0)$. A circular wire is placed in the near wake of the main cylinder at $(x / D, y / D)=(0.75,0.75)$, to be consistent with the 
experimental and numerical setups in Zhang et al. (1995) and Yildirim et al. (2013a,b). For the majority of the present study, the diameter of the wire, $D_{w}$, is chosen as $0.05 D$, which is the same as that employed in the numerical study by Zhang et al. (1995) but larger than those used in the experiments: $D_{w}=0.006 D$ in Zhang et al. (1995) and $D_{w}$ $=0.01 \mathrm{D}$ in Yildirim et al. $(2013 \mathrm{a}, \mathrm{b})$. This is because the use of a finer-scale wire would result in a much larger computational cost due to the requirement of resolving the flow field close to the wire. For example, the computational cost is increased by 2 to 3 times when $D_{w}$ is reduced from $0.05 D$ to $0.01 D$. Nevertheless, a $D_{w}$ of $0.01 D$ is also adopted for some cases in section 3.1.1 to investigate the influence of $D_{w}$ on the onset of the Mode $\mathrm{C}$ wake instability.

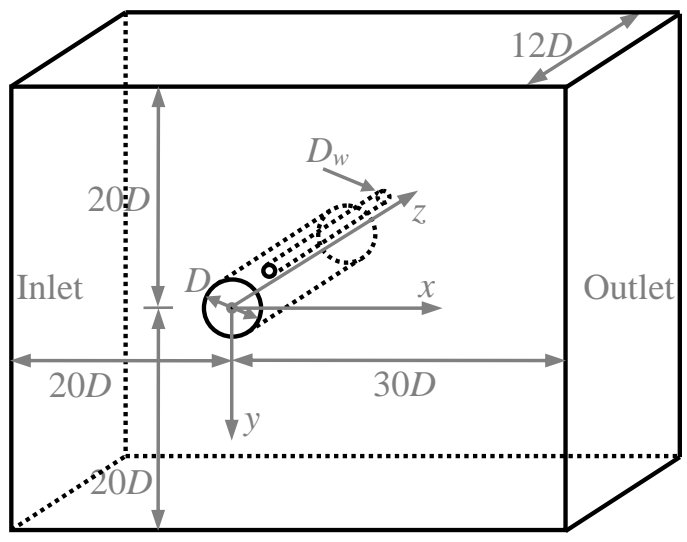

(a)

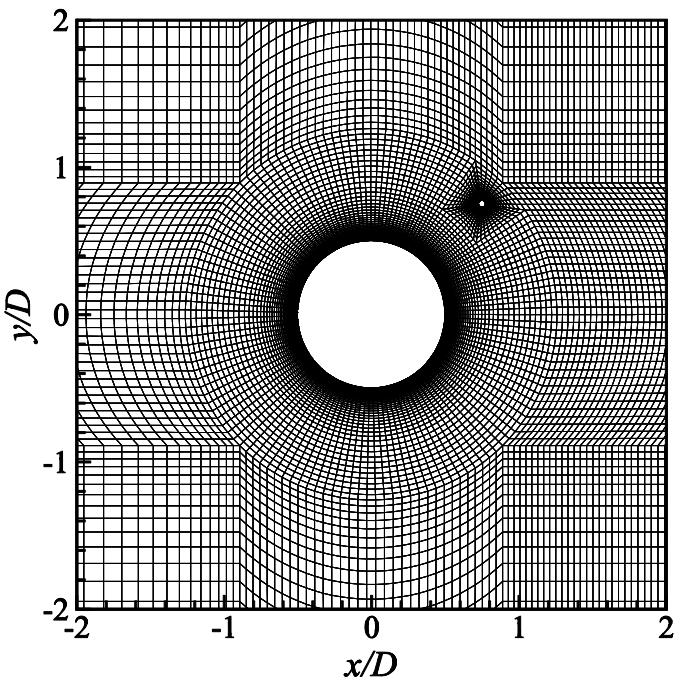

(b)

Fig. 1. (a) Schematic model of the computational domain (not to scale), and (b) Close-up view of the 2D standard mesh near the main cylinder and wire.

The boundary conditions are specified as follows. At the inlet, a uniform flow velocity $U$ is specified in the $x$-direction. At the outlet, the Neumann boundary condition (i.e. zero normal gradient) is applied for the velocity, and the pressure is specified as a reference value of zero. Symmetry boundary conditions are applied at the top and bottom boundaries, while periodic boundary conditions are employed at the two lateral boundaries perpendicular to the cylinder axis. A no-slip condition is 
applied on the surfaces of the main cylinder and wire.

Periodic boundary conditions have been routinely employed in numerical simulations to approximate the flow past bluff bodies of an infinite spanwise length (e.g. Karniadakis and Triantafyllou, 1992; Henderson, 1997; Braza et al., 2001; Lei et al., 2001). For the case of an isolated circular cylinder, Henderson (1997) suggested that a moderate spanwise domain length $L_{z}(\sim 10 D)$ is sufficient for deducing what happens in the limit $L_{z} \rightarrow \infty$, while a small system of $L_{z} \sim 1 D$ may give rise to an inaccurate time-periodic flow behaviour. Similarly, Jiang et al. (2017b) recommended an $L_{z}$ of approximately three times the intrinsic wavelength of the mode, since a smaller $L_{z}$ would restrict the spanwise distribution of the vortices to inaccurate patterns. For the present Mode $\mathrm{C}$ flows, the use of $L_{z}=12 \mathrm{D}$ accommodates more than six spanwise periods of Mode C. The adequacy of $L_{z}=12 \mathrm{D}$ is further demonstrated by examining the statistically stationary streamwise enstrophy (a quantification of the flow three-dimensionality) under different selections of $L_{z}$. The streamwise enstrophy $\varepsilon_{x}$ is defined as:

$\varepsilon_{x}=\frac{1}{2} \int_{V} \omega_{x}^{2} \mathrm{~d} V$

$\omega_{x}=\left(\frac{\partial u_{z}}{\partial y}-\frac{\partial u_{y}}{\partial z}\right) \frac{D}{U}$

where $V$ is the volume of the flow field of interest, which is the near-wake region of $x / D=0-10$ and the entire lengths in the $y$-and $z$-directions for the present study. Fig. 2 shows the influence of $L_{z}$ on the streamwise enstrophy per unit span length $\left(\varepsilon_{x} / L_{z}\right)$ for $R e=175,200$ and 280. The convergence of $\varepsilon_{x} / L_{z}$ at $L_{z} \geq 12 D$ suggests that an $L_{z}$ of $12 D$ is adequate. 


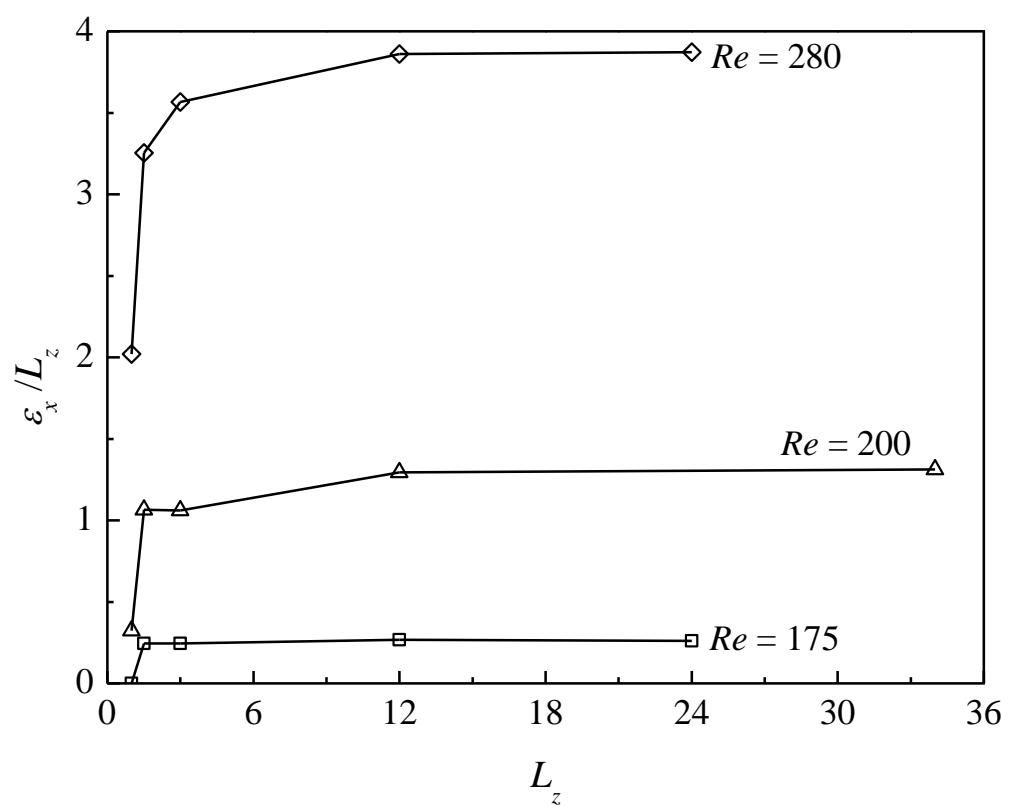

Fig. 2. Influence of the spanwise domain length $L_{z}$ on the streamwise enstrophy per unit span length.

\subsection{Mesh convergence}

The computational domain size and mesh resolution have been chosen based on a mesh convergence check, which has been carried out in two steps: (i) The adequacy of domain size and mesh resolution in the $x-y$ plane (i.e. the plane perpendicular to the cylinder span) was examined by a set of $2 \mathrm{D}$ simulations. (ii) A 3D mesh was then generated by replicating the selected 2D mesh along the $z$-axis. A mesh dependence study on this 3D mesh was then undertaken. The selected $2 \mathrm{D}$ mesh and the corresponding 3D mesh are referred to as the standard meshes throughout.

The standard 2D mesh (Fig. 1b) is detailed as follows. The computational domain size in the $x-y$ plane is $50 D \times 40 D$. For the main cylinder, the cylinder perimeter is discretized with 142 nodes, and the radial size of the first layer of mesh next to the cylinder is $0.001 D$. For the near-wake wire with a diameter $D_{w}$ of $0.05 D$ or $0.01 D$, the wire perimeter is discretized with 42 nodes, and the radial size of the first layer of mesh next to the wire is $0.0054 D_{w}$. The non-dimensional wall distances $y^{+}$on the main cylinder and wire surfaces are less than 0.1 for all the cases considered in this study (up to $R e=400$ ). A relatively high mesh resolution is used in the near wake by 
specifying a streamwise cell size varying linearly from $0.04 D$ at $x / D=1$ to $0.09 D$ at $x / D=10$.

The time step $\Delta t$ is chosen based on a Courant-Friedrichs-Lewy (CFL) limit of 0.5 , where the CFL number is defined as:

$\mathrm{CFL}=\frac{|u| \Delta t}{\Delta l}$

where $|u|$ is the magnitude of the velocity through a cell, and $\Delta l$ is the cell size in the direction of the velocity.

The adequacy of mesh resolution and domain size of the standard 2D mesh was checked at $R e=400$ (the largest $R e$ adopted in this study). The influence of the mesh resolution and domain size is quantified by calculating the hydrodynamic forces on the main cylinder and wire, including the Strouhal number $(S t)$ and the drag and lift coefficients $\left(C_{D}\right.$ and $\left.C_{L}\right)$, which are defined as:

$$
\begin{aligned}
& S t=f_{L} \frac{D}{U} \\
& C_{D}=\frac{F_{D}}{\frac{1}{2} \rho U^{2} D L_{z}} \\
& C_{L}=\frac{F_{L}}{\frac{1}{2} \rho U^{2} D L_{z}}
\end{aligned}
$$

where $f_{L}$ is the frequency of the fluctuating lift force, and $F_{D}$ and $F_{L}$ are the integrated drag and lift forces on the cylinder, respectively. The time-averaged drag and lift coefficients are denoted as $\overline{C_{D}}$ and $\overline{C_{L}}$, respectively. The root-mean-square lift coefficient $C_{L}{ }^{\prime}$ is defined as:

$$
C_{L}^{\prime}=\sqrt{\frac{1}{N} \sum_{i=1}^{N}\left(C_{L, i}-\overline{C_{L}}\right)^{2}}
$$

where $N$ is the number of values in the time history of $C_{L}$. It is worth noting that the frequencies of the fluctuating forces on the main cylinder and near-wake wire are naturally identical. This is because for $R e \leq 400$ the Reynolds number based on the wire diameter $\operatorname{Re}_{w}\left(=U D_{w} / v\right)$ is smaller than 20 , much less than the threshold of 
natural vortex shedding of approximately 47 (e.g. Henderson, 1997).

The 2D mesh dependence study shows that by doubling the domain size from the cylinder centre to either the inlet, the outlet, or the transverse boundaries, or by doubling the amount of cells in both the $x$ - and $y$-directions, the relative differences in the $S t, \overline{C_{D}}$ and $C_{L}{ }^{\prime}$ values on the main cylinder are within $1 \%$, and those on the near-wake wire are within $1.5 \%$, which suggests that the standard $2 \mathrm{D}$ mesh is adequate for the present study.

The 3D mesh dependence study focused on the mesh resolution and domain size in the spanwise direction. For the standard 3D mesh, the spanwise domain length $\left(L_{z}\right)$ is $12 D$ and the cell size in the spanwise direction $(\Delta z)$ is $0.1 D$. To examine the influence of $L_{z}$ and $\Delta z$, an extended mesh (with $L_{z}$ increased from $12 D$ to $24 D$ ) and a refined mesh (with $\Delta z$ decreased from $0.1 D$ to $0.05 D$ ) were tested at $R e=400$. Since the fully developed 3D flows may be irregular, at least 800 non-dimensional time units (defined as $t^{*}=t U / D$ ) of the fully developed flow were used to calculate the statistical stationary $S t$ and $\overline{C_{D}}$ (for which the results calculated from the second half of the sampled data are within $0.5 \%$ of those determined from the entire sampled data sets). For the 3D analysis, $S t$ is determined using the peak frequency derived from the fast Fourier transform (FFT) of the time history of the lift coefficient.

As shown in Table 1, the numerical results calculated with the extended and refined meshes are very close to those calculated with the standard 3D mesh (relative differences are within $0.3 \%$ ). With regard to the flow field in the near wake, Fig. 3 shows the time- and span-averaged streamwise and transverse velocity profiles at a few streamwise locations $(x / D=1,2,3$, and 5) calculated with the three meshes listed in Table 1 at $R e=400$. Good agreements are observed in Fig. 3 for the averaged velocity profiles, including at around $(x / D, y / D)=(1,0.75)$ where fluctuations of the velocity profiles are observed due to the presence of the wire.

Based on the mesh dependence study reported above, the standard 3D mesh is considered to be adequate and is used for the 3D simulations of the present study. 
Table 1. Results of the 3D mesh dependence study at $R e=400$. The results of Cases 2 and 3 are shown by the relative differences with respect to Case 1 .

\begin{tabular}{llllllll}
\hline Case & Mesh type & $L_{z} / D$ & $\Delta z / D$ & \multicolumn{2}{c}{ Main cylinder } & \multicolumn{2}{l}{ Near-wake wire } \\
\cline { 5 - 8 } & & & & $S t$ & $\overline{C_{D}}$ & $S t$ & $\overline{C_{D}}$ \\
\hline 1 & Standard & 12 & 0.1 & 0.1785 & 1.0522 & 0.008928 & 2.5883 \\
2 & Extended & 24 & 0.1 & $+0.17 \%$ & $+0.23 \%$ & $+0.17 \%$ & $+0.21 \%$ \\
3 & Refined & 12 & 0.05 & $+0.08 \%$ & $-0.01 \%$ & $+0.08 \%$ & $-0.13 \%$ \\
\hline
\end{tabular}

(a)

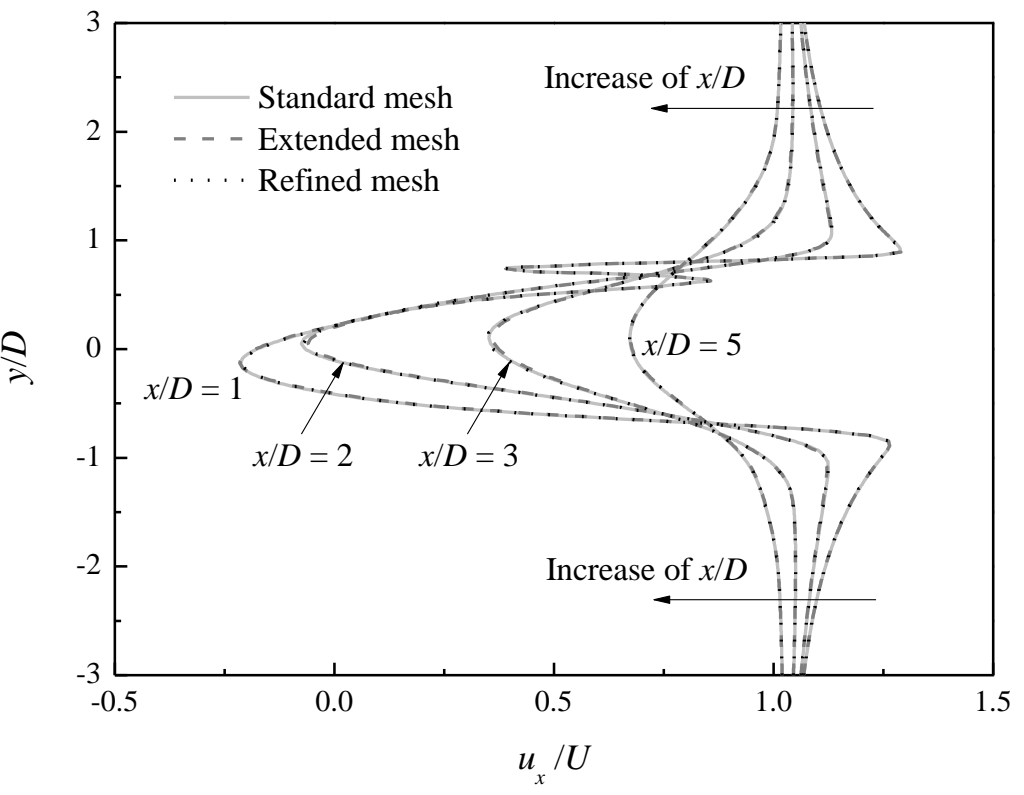

(b)

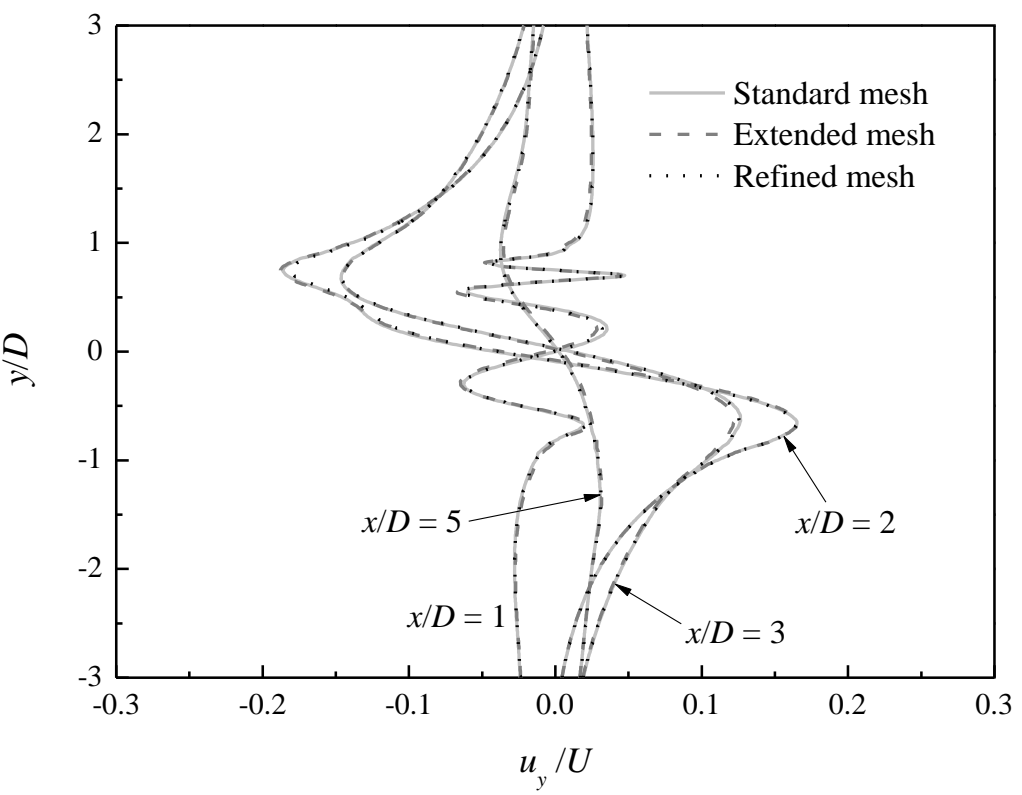

Fig. 3. Time- and span-averaged velocity profiles at a few streamwise locations in the 
near wake $(x / D=1,2,3$, and 5) calculated with the three meshes listed in Table 1 at $R e=400:$ (a) streamwise velocity profiles, and (b) transverse velocity profiles.

\section{Numerical results}

\subsection{Onset of the Mode C instability}

\subsubsection{Neutral instability curve}

As reported in Zhang et al. (1995) and Yildirim et al. (2013a,b), the secondary instability of flow past a circular cylinder with a near-wake wire disturbance is represented by a Mode $\mathrm{C}$ instability. To complement previous studies, the neutral instability curves for the Mode $\mathrm{C}$ instability based on two different wire diameters $\left(D_{w} / D=0.05\right.$ and 0.01$)$ are calculated in this study through 3D DNS, and the results are shown in Fig. 4. Each point on the neutral instability curve is obtained by specifying a fixed spanwise domain length $L_{z}$ (i.e. a fixed spanwise wavelength $\lambda$ ) and varying $R e$ so that the critical $R e$ corresponding to the prescribed $\lambda$ is determined. The point at the left tip of the neutral curve is the critical $R e\left(R_{c r}\right)$ for the onset of the Mode C instability. More details on this method can be found in Jiang et al. (2017a,c). The computational cost of this method is similar to that of Floquet stability analysis, since only a half of a spanwise period of the Mode C structure is simulated (by using $L_{z}=\lambda / 2, L_{z} / \Delta z=5$ and symmetry boundary conditions at the two lateral boundaries to isolate a half of a spanwise period of Mode C). It has been checked that based on the reference case with $D_{w} / D=0.05$ and $L_{z} / D=0.75$ (i.e. $\lambda / D=1.5$ ) which is located at the left tip of the neutral curve shown in Fig. 4, the variations of $R e_{c r}$ after doubling the domain size in the $x-y$ plane (to $100 D \times 80 D$ ), doubling the cell numbers in both the $x$ - and $y$-directions, or doubling the mesh layers in the spanwise direction (to $L z / \Delta z=$ 10) are all within $0.7 \%$. 


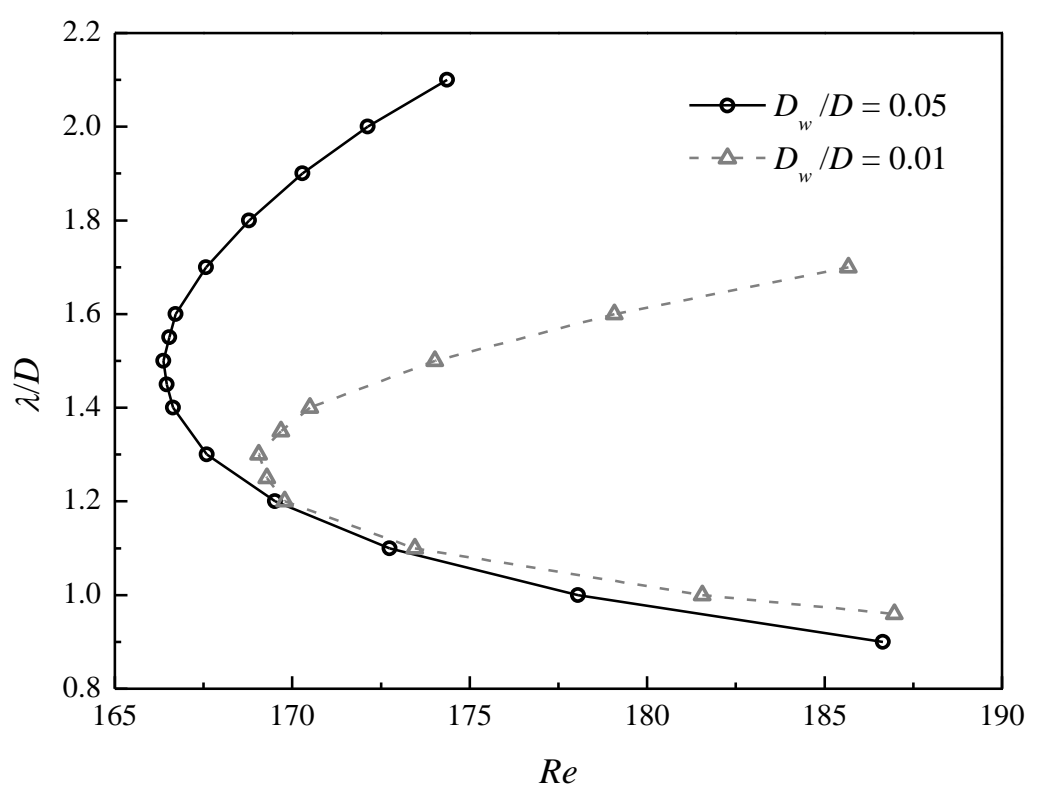

Fig. 4. Neutral instability curves for the Mode $\mathrm{C}$ instability based on two different wire diameters.

As shown in Fig. 4, the neutral curve for the Mode C instability varies with the variation of $D_{w}$. The $R e_{c r}$ value at the left tip of the neutral curve and the corresponding $\lambda_{c r}$ for Mode $\mathrm{C}$ vary slightly from $\left(R e_{c r}, \lambda_{c r} / D\right)=(166.4,1.5)$ for $D_{w} / D$ $=0.05$ to $\left(\operatorname{Re}_{c r}, \lambda_{c r} / D\right)=(169.1,1.3)$ for $D_{w} / D=0.01$. The $R e_{c r}$ values agree well with the experimental results reported in the literature, e.g. $R e_{c r}=170$ by Zhang et al. (1995) with $D_{w} / D=0.006$, and $R e_{c r}=165-180$ by Yildirim et al. (2013a) with $D_{w} / D$ $=0.01$.

The $\lambda_{c r}$ values represent the spanwise wavelengths at the onset of Mode $\mathrm{C}$ and are smaller than $\lambda \sim 2 D$ observed in the experimental studies of Zhang et al. (1995) and Yildirim et al. (2013a,b) over the range of $R e \sim 170-270$. On the other hand, full 3D DNS at $R e=210$ produces uniform Mode $\mathrm{C}$ structures with $\lambda \sim 1.7 D$, which is similar to the numerical result of $\lambda \sim 1.8 D$ in Zhang et al. (1995). The above results indicate that in the regime of uniform Mode $\mathrm{C}, \lambda$ increases with increasing $R e$.

\subsubsection{Floquet analysis of the wake modes}

In addition to the DNS results of the neutral instability curves of Mode $\mathrm{C}$ reported 
in section 3.1.1, Floquet stability analysis is also conducted for the scenario of $D_{w} / D=$ 0.05 to examine the possibility of additional unstable and stable wake modes other than Mode C. The methodology of Floquet analysis can be found in Barkley and Henderson (1996) and is omitted here. An open-source code, Nektar++ (Cantwell et al., 2015), is used for the Floquet analysis. As a validation, the present Floquet analysis predicts an $\operatorname{Re}_{\mathrm{c} r}$ of 189.8 for the onset of Mode A for flow past a circular cylinder, which is in a good agreement with that of $190.2( \pm 0.02)$ in Posdziech and Grundmann (2001) and $190( \pm 1)$ in Carmo et al. (2008).

For the scenario of $D_{w} / D=0.05$, the dependence of the dominant Floquet multiplier $\mu$ on the spanwise wavenumber $\beta(=2 \pi / \lambda)$ for various $R e$ values is shown in Fig. 5. For each $R e$, only a single peak region is observed, and the dominant Floquet multipliers in the peak region are real and negative, which corresponds to the subharmonic Mode C (Carmo et al., 2008; Sheard et al., 2009). This single-peak feature is different from a number of other Mode $\mathrm{C}$ scenarios mentioned in section 1, e.g. a circular ring (Sheard et al., 2003; 2005b), two circular cylinders in staggered arrangements (Carmo et al., 2008), a rotated square cylinder (Sheard et al., 2009), a circular cylinder subjected to planar shear flow (Park and Yang, 2018), etc., for which multiple peak regions exist in the $|\mu|-\beta$ space, indicating the wake is unstable to multiple modes including not only Mode $\mathrm{C}$ but also Modes A, B, etc. Even when Mode $\mathrm{C}$ is the first 3D wake instability mode, the Mode $\mathrm{C}$ wake structures may be replaced by other wake structures when other wake modes become unstable at higher $R e$ values (e.g. Sheard et al., 2005b). In contrast, for the present scenario, the single-peak feature allows a "clean" Mode $\mathrm{C}$ to evolve with time and $R e$ (to be examined in section 3.2) without potential interactions with other wake modes. 


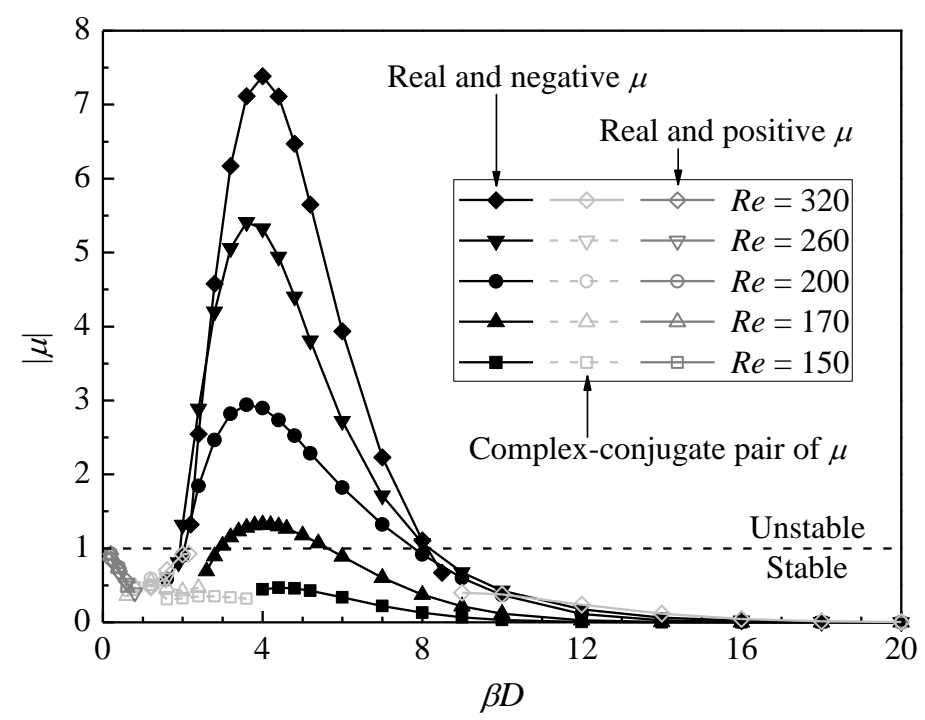

Fig. 5. Dependence of the dominant Floquet multiplier $\mu$ on the spanwise wavenumber $\beta(=2 \pi / \lambda)$ for the scenario of $D_{w} / D=0.05$. The horizontal dashed line shows the neutral instability threshold at $|\mu|=1$.

\subsubsection{Hysteresis of the Mode $C$ instability}

Depending on the type and spatial arrangement of the bluff bodies, both subcritical (hysteretic) and supercritical (non-hysteretic) Mode C instabilities have been observed in the literature (e.g. Sheard et al., 2004; Carmo et al., 2008). For the present scenario, the existence of hysteresis at the onset of the Mode $\mathrm{C}$ instability is examined by using the Landau model (Landau and Lifshitz, 1976). The Landau equation, which considers the amplitude $A(t)$ of a perturbation mode, is written up to third order as:

$\frac{\mathrm{d} A}{\mathrm{~d} t}=(\sigma+\mathrm{i} \omega) A-l(1+\mathrm{i} c)|A|^{2} A+\ldots$

where $\sigma$ is the linear growth rate of the perturbation, $\omega$ is the angular oscillation frequency during the linear growth phase, and $c$ is the Landau constant. The $l$-coefficient is determined by plotting $(\mathrm{d} \log |A| / \mathrm{d} t)$ against $|A|^{2}$, where the slope of the curve near $|A|^{2}=0$ gives $-l$. The hysteresis of the wake instability mode is determined based on the sign of the $l$-coefficient. A positive $l$ corresponds to a non-hysteretic flow, while a negative $l$ corresponds to a hysteretic flow. More details on this method can be 
found in Dušek et al. (1994), Sheard et al. (2004), and Carmo et al. (2008).

The Landau model is applied on the case with $R e=167$ (slightly above $R e_{c r}$ of $166.4)$ and $L_{z}=1.5 D\left(=\lambda_{c r}\right)$. The mode amplitude $|A|$ is measured from the envelope of the oscillation of the spanwise velocity component sampled at a point in the wake $(x / D, y / D, z / D)=(3,1,0.6)$. The relationship between $(\mathrm{d} \log |A| / \mathrm{d} t)$ and $|A|^{2}$ (omitted for simplicity) gives a positive $l$-coefficient near $|A|^{2}=0$, which suggests that the present Mode $\mathrm{C}$ instability is non-hysteretic.

The non-hysteretic Mode $\mathrm{C}$ instability is further confirmed by adopting the fully developed Mode C flow (calculated by DNS with $L_{z}=12 D$ ) at $R e=166.5$ (slightly above $R e_{c r}$ ) as the initial condition and reducing $R e$ by a small interval each time until the Mode $\mathrm{C}$ structure disappears naturally. The first attempt is to reduce $R e$ from 166.5 to 165.5 . The use of an interval of $R e$ of 1.0 is because the flow decays extremely slowly at $R e$ very close to $R e_{c r}$. With the evolution over time, the Mode $\mathrm{C}$ structure becomes less well-defined at $t^{*} \sim 900$ and disappears completely at $t^{*} \sim$ 1900. This case further suggests that there is no hysteresis effect for the present Mode $\mathrm{C}$ instability and thus the transition to Mode $\mathrm{C}$ is supercritical.

\subsection{Three-dimensional wake transition}

In this section, the evolution of the Mode $\mathrm{C}$ flow structure over the process of wake transition to chaos is examined with a near-wake wire of $D_{w} / D=0.05$ through 3D DNS. Three flow regimes are observed with increase in $R e$, namely the uniform periodic regime $\left(R e=R e_{c r}-210\right)$, the non-uniform periodic regime $(R e=220-230)$, and the chaotic regime $(R e \geq 240)$. Here "uniform" and "non-uniform" refer to the repeatability of the Mode $\mathrm{C}$ structure along the spanwise direction, while "periodic" refers to the repeatability of the Mode $\mathrm{C}$ structure over time.

\subsubsection{Uniform periodic regime for $R e=R e_{c r}-210$}

For $R e=\operatorname{Re}_{c r}-210$, the fully developed flow structure is represented by a 
well-defined Mode $\mathrm{C}$ structure with a uniform spanwise wavelength and a $2 T$-periodicity (e.g. Fig. 6). The vortex structures are captured by the iso-surfaces of the streamwise vorticity $\omega_{x}$. As shown in Fig. 6, every two adjacent streamwise vortices with opposite signs form a streamwise vortex pair. The spatiotemporal structure of the vortices can be described by a spatiotemporal symmetry expressed as:

$$
\omega_{x}(x, y, z, t)=\omega_{x}(x, y, z \pm \lambda / 2, t+T)
$$

It is noted that while the spatiotemporal structure of the vortices is in the form of equation (3.2), the streamwise vortices are not indeed shifted along the spanwise direction by $\lambda / 2$ over each $T$ (in a travelling wave form). It is confirmed through visualisation of 40 snapshots over $2 T$ that each streamwise vortex remains at its own spanwise location as it propagates downstream (in a standing wave form).
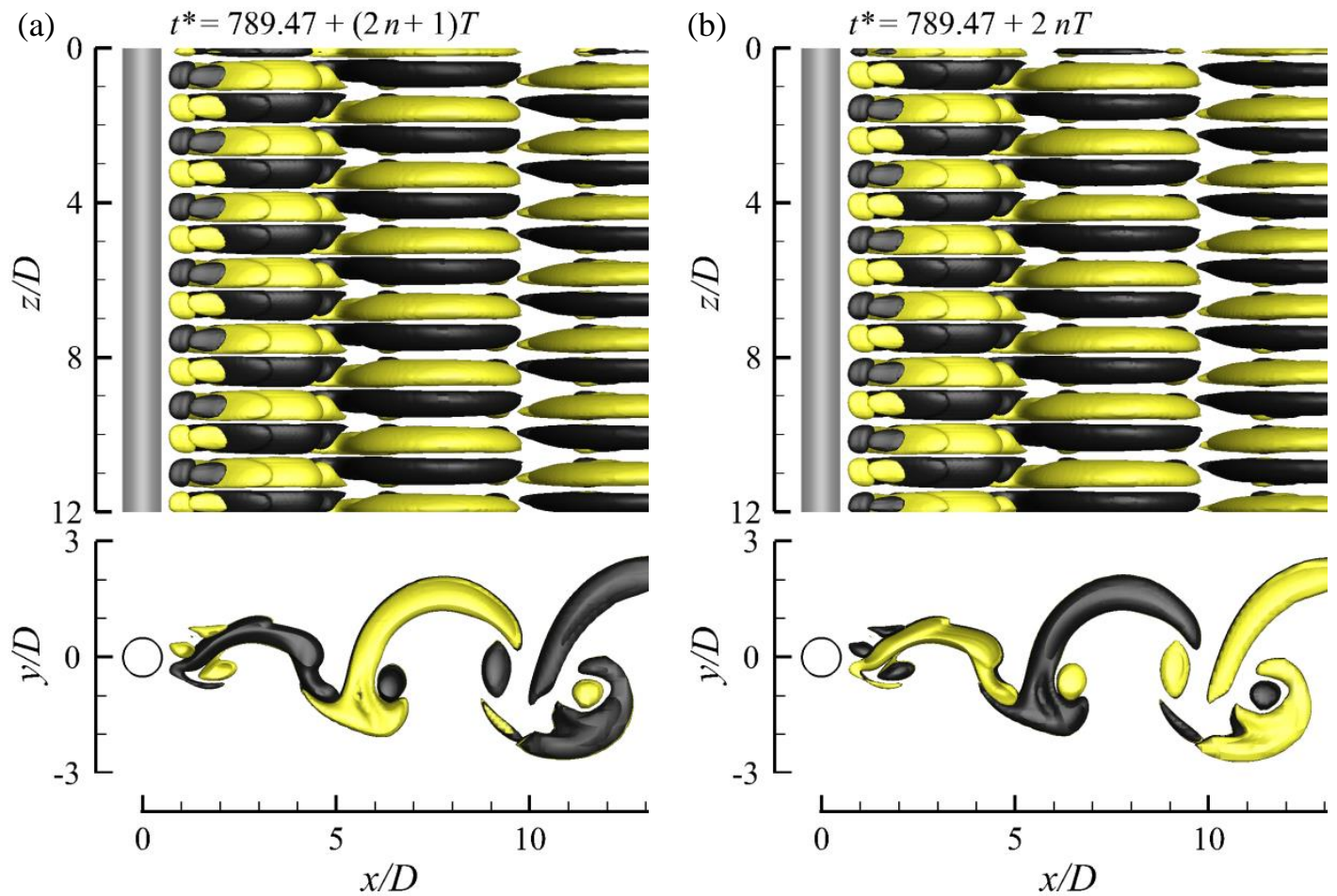

Fig. 6. Mode $\mathrm{C}$ structures at $R e=210$ captured by the iso-surfaces of $\omega_{x}= \pm 0$.2. Dark grey and light yellow denote positive and negative values, respectively. The flow is from left to right past the cylinder on the left. The Mode $\mathrm{C}$ structures are $2 T$-periodic and are therefore shown in panels (a) and (b) at $1 T$ apart. Each graph is shown at the time instant when the lift coefficient on the main cylinder reaches a local maximum. 
In the expression of $t^{*}, n$ denotes an integer.

Fig. 7 shows the time histories of the lift coefficient for $R e=210-240$. The entire ranges of the time histories are shown in the left column, while the time histories of 50 non-dimensional time units of the fully developed flow near the end of each simulation are enlarged in the right column. The periodicity of the drag coefficient is the same as that of the lift coefficient and is omitted for simplicity. For $R e=167-210$ (e.g. Fig. 7a), the time histories of the lift coefficient are almost $T$-periodic rather than $2 T$-periodic. This is because the force coefficients are calculated through integration over the entire spanwise length. For example, the two flow fields of $1 T$ apart shown in Fig. 6 both contain seven uniform Mode C streamwise vortex pairs. Therefore, the force coefficients that are averaged over the seven pairs of Mode $\mathrm{C}$ are repeatable between two consecutive vortex shedding periods.

To uncover the $2 T$-periodic nature of Mode $\mathrm{C}$ that is not revealed by the integrated force coefficients, time histories of the velocity components sampled at a near-wake point $(x / D, y / D, z / D)=(3,1,6)$ are examined. Since the periodicity of the three velocity components is the same, only the time history of $u_{y}$ is shown in Fig. 8 . For $R e=167-210$, although the time histories of the force coefficients are almost $T$-periodic (e.g. Fig. 7a), the time histories of the point velocity components are clearly $2 T$-periodic (e.g. Fig. 8a). 

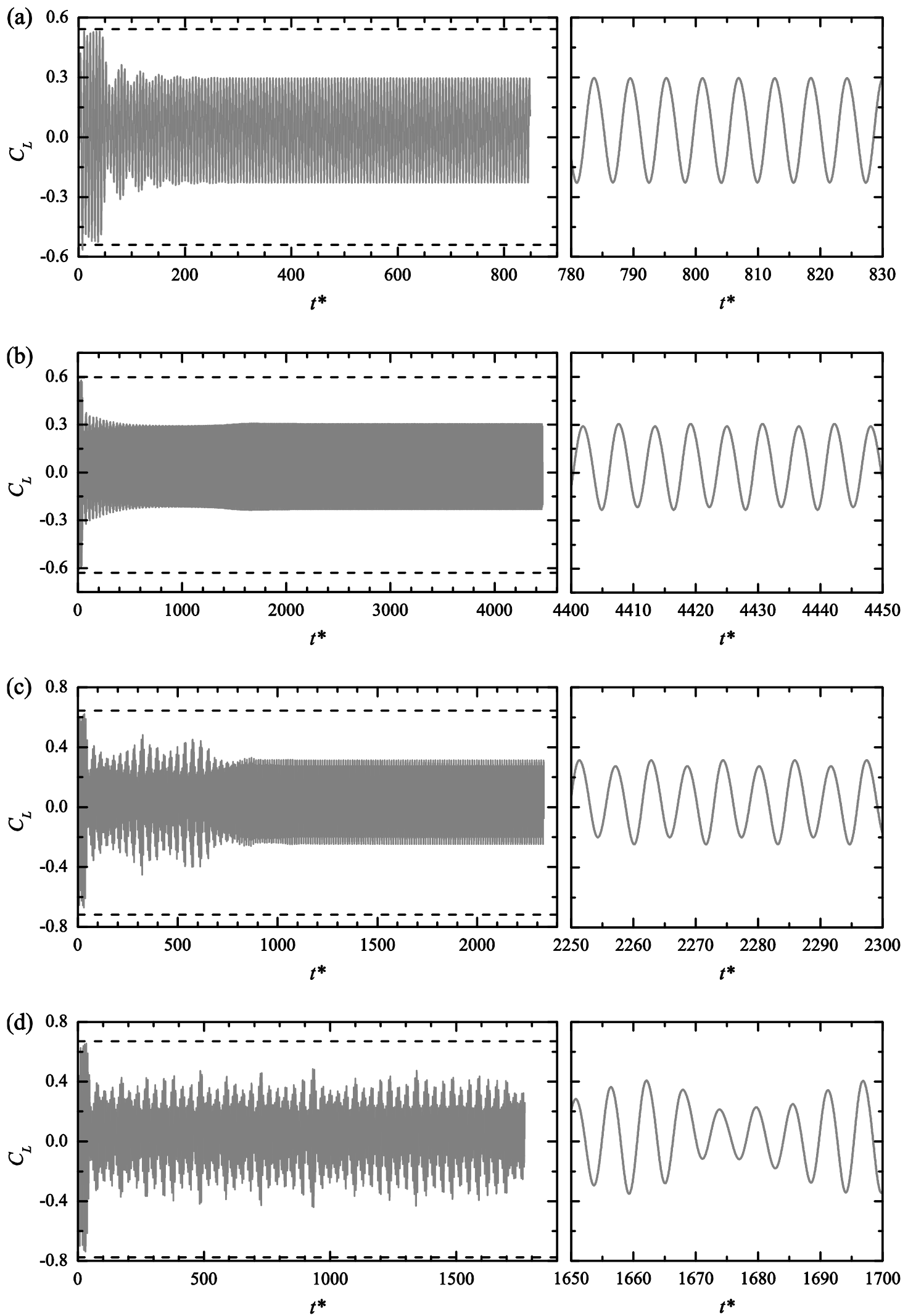

Fig. 7. Time histories of the lift coefficient for (a) $R e=210$, (b) $R e=220$, (c) $R e=$ 230, and (d) $R e=240$. The horizontal dashed lines in the left column of graphs mark the fluctuation range of the corresponding 2D lift coefficient. 

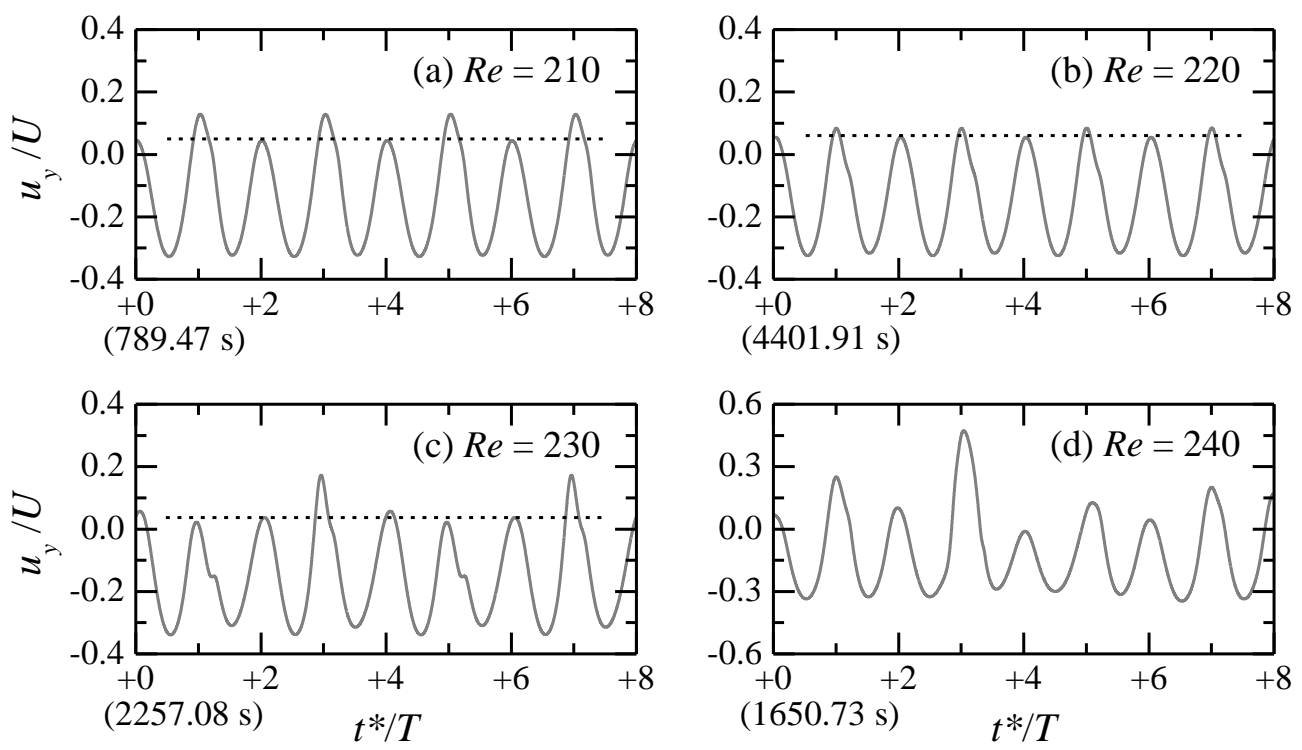

Fig. 8. Time history of $u_{y}$ sampled at $(x / D, y / D, z / D)=(3,1,6)$ for (a) $R e=210$, (b) $R e=220,(\mathrm{c}) \operatorname{Re}=230$, and (d) $R e=240$. The horizontal dashed lines are used to facilitate visualisation of the periodicity of the time histories. For each $R e, t^{*}=n T$ are the time instants when the lift coefficient on the main cylinder reaches a local maximum, and correspond to the Mode $\mathrm{C}$ structures shown in Fig. 6, Fig. 9, Fig. 10 and Fig. 11.

\subsubsection{Non-uniform periodic regime for $R e=220-230$}

As $R e$ increases from 210 to 220, well-defined Mode C structure similar to those shown in Fig. 6 only persists for a short period of time (at $t^{*} \lesssim 1200$ ) before it evolves spontaneously into a more stable pattern of non-uniform Mode C. The fully developed non-uniform Mode $\mathrm{C}$ structures at $R e=220$ are shown in Fig. 9. The evolution of the Mode C pattern at $t^{*} \sim 1200$ is also reflected by the corresponding change in the pattern of the time history of the lift coefficient shown in Fig. 7(b). The evolution of the Mode C pattern resembles the evolution from Mode A to Mode A with vortex dislocations for an isolated circular cylinder (Williamson, 1996), which is likely due to an uneven growth in strength of some particular streamwise vortices over time. 

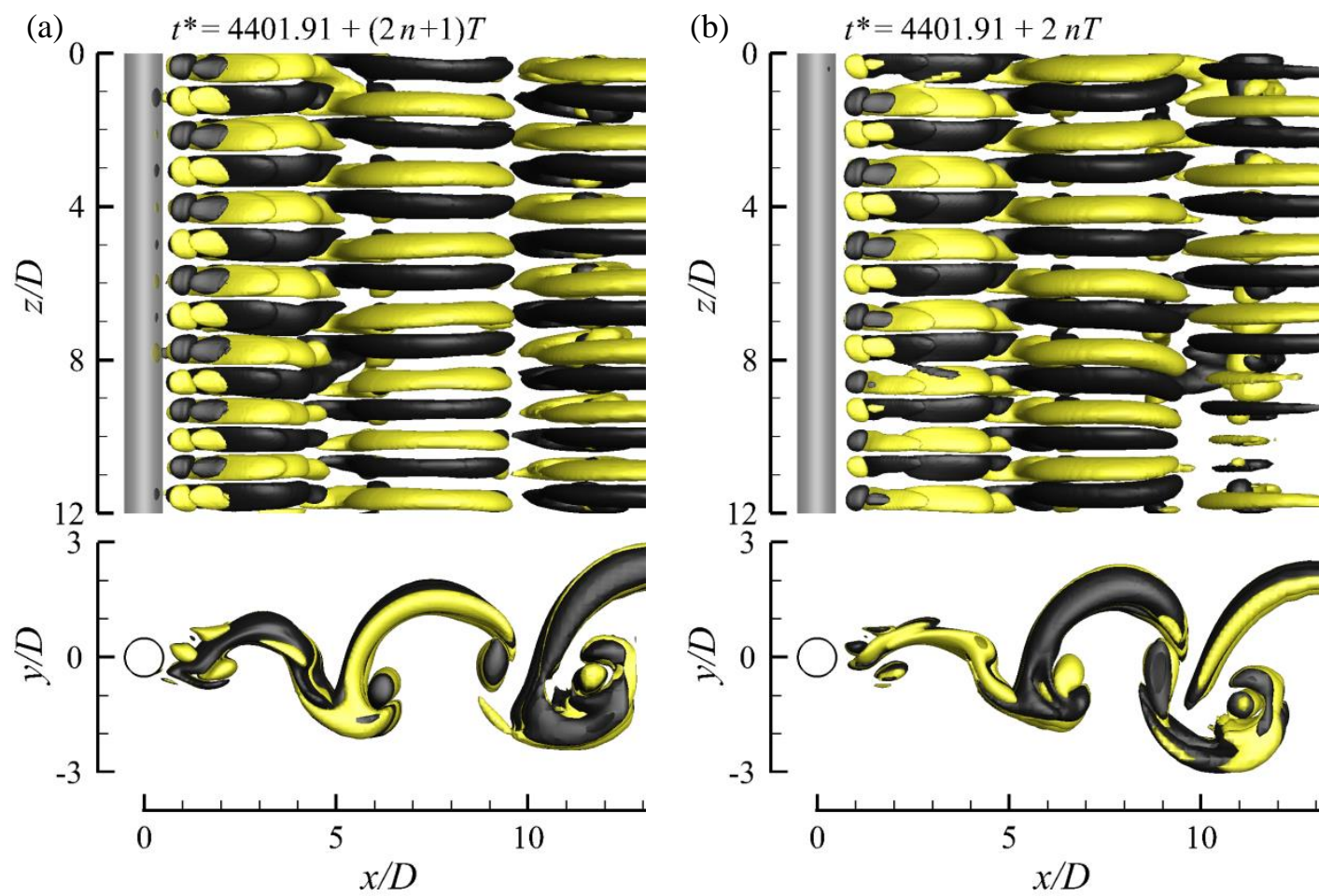

Fig. 9. Mode $\mathrm{C}$ structures at $R e=220$ captured by the iso-surfaces of $\omega_{x}= \pm 0.25$. The Mode $\mathrm{C}$ structures are 2T-periodic and are therefore shown in panels (a) and (b) at $1 T$ apart. The spanwise wavelengths shown in the $x-z$ plane are slightly varied, e.g. the spanwise wavelengths at $z / D \sim 8-12$ are smaller than those at $z / D \sim 0-8$.

When the Mode $\mathrm{C}$ structure first becomes non-uniform at $R e=220$, the degree of uneven growth in strength and the degree of non-uniformity of the streamwise vortices are small, such that the fully developed Mode C structure still maintains the self-sustaining of streamwise vortices with no further uneven growth in strength and remains strictly $2 T$-periodic (Fig. 8b). In addition, due to the loss of the spatiotemporal symmetry described in equation (3.2), the time histories of the force coefficients (e.g. Fig. 7b) become $2 T$-periodic.

At $R e=230$, the increasingly non-uniform Mode $\mathrm{C}$ flow can no longer remain $2 T$-periodic and evolves to a $4 T$-periodicity (Fig. 10). It is worth noting that the temporal evolution from the well-defined Mode C structure to the non-uniform Mode $\mathrm{C}$ for $R e=220$ and 230 and the subsequent period quadrupling at $R e=230$ are new phenomena that have not been reported previously, possibly due to a relatively long 
temporal evolution required for a fully developed flow (see Fig. 7b,c).
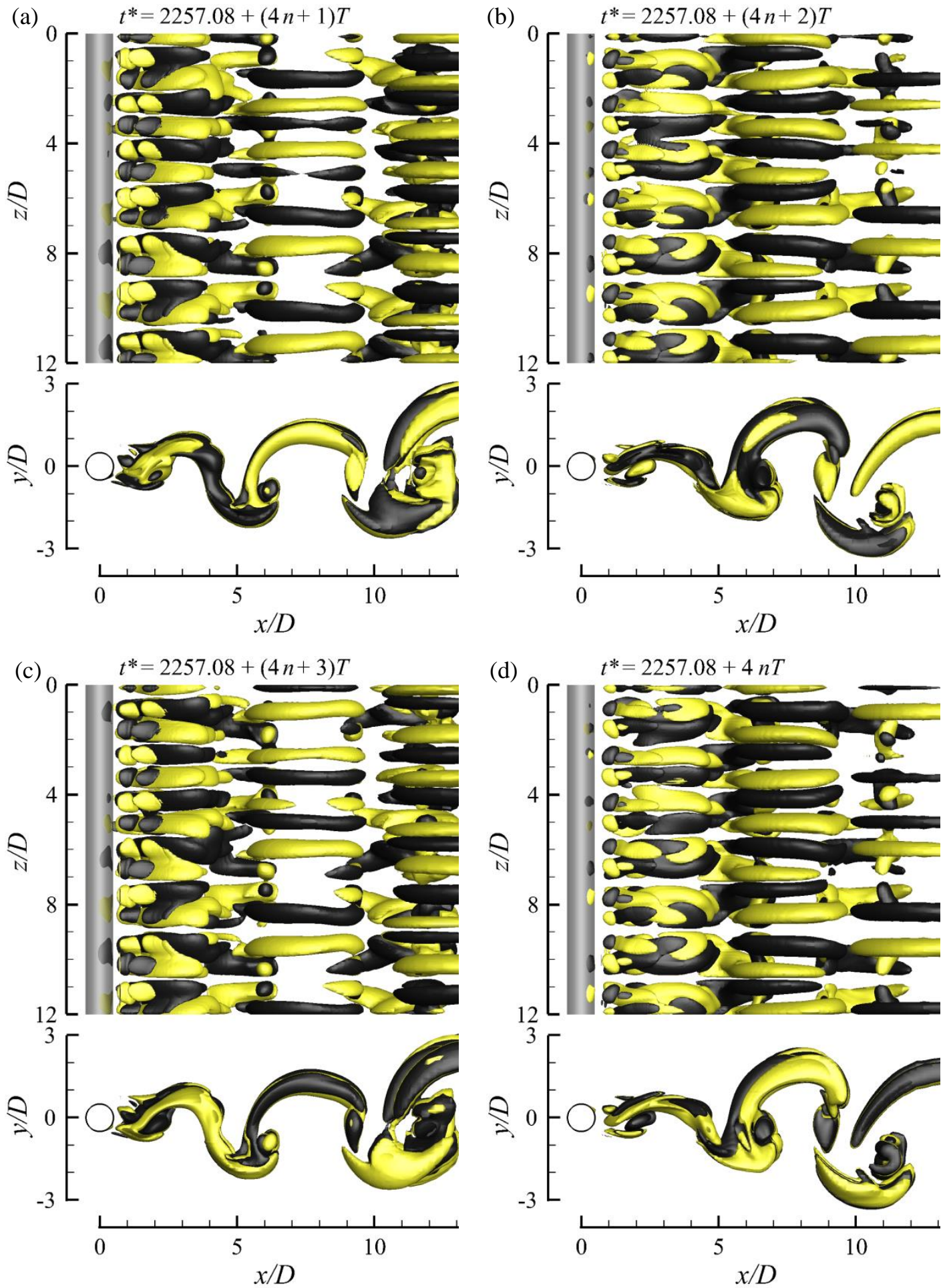

Fig. 10. Mode $\mathrm{C}$ structures at $R e=230$ captured by the iso-surfaces of $\omega_{x}= \pm 0.3$. The Mode C structures are 4T-periodic and are therefore shown in panels (a) to (d) at $1 T$ apart. 
At $R e=230$, the streamwise vortices at respective spanwise locations still change signs after each vortex shedding period. However, after $2 T$ within which the signs of the streamwise vortices have changed twice, the flow structure does not repeat itself (Fig. 10). Nevertheless, the flow structures $2 T$ apart resemble each other while the flow structures $1 T$ apart are much more different (Fig. 10). For example, the flow structure shown in Fig. 10(c) resembles that shown in Fig. 10(a) after a spanwise shift of approximately $2 D$. Therefore, the time histories of the integrated force coefficients are $2 T$-periodic (Fig. 7c), while the time histories of the velocity components sampled at a fixed point are $4 T$-periodic (Fig. 8c). The time histories of the point velocity components are a better indication than the time histories of the force coefficients to quantify the periodicity of the flow.

\subsubsection{Chaotic regime for $R e \geq 240$}

For $R e \geq 240$, the increasingly non-uniform Mode C flow loses periodicity. For example, the time histories of the force coefficients (Fig. 7d) and point velocity components (Fig. 8d) for $R e=240$ are no longer highly repeatable in a finite number of vortex shedding cycles. In particular, the Mode C structures at $R e=240$ of $4 T$ apart shown in Fig. 11 look quite different, and the point velocity signal at the corresponding time instants shows local peaks of quite different amplitudes (Fig. 8d). For the latter time instant (Fig. 11b), the weaker Mode C structure at the sample point results in a smaller velocity peak shown in Fig. 8(d). 

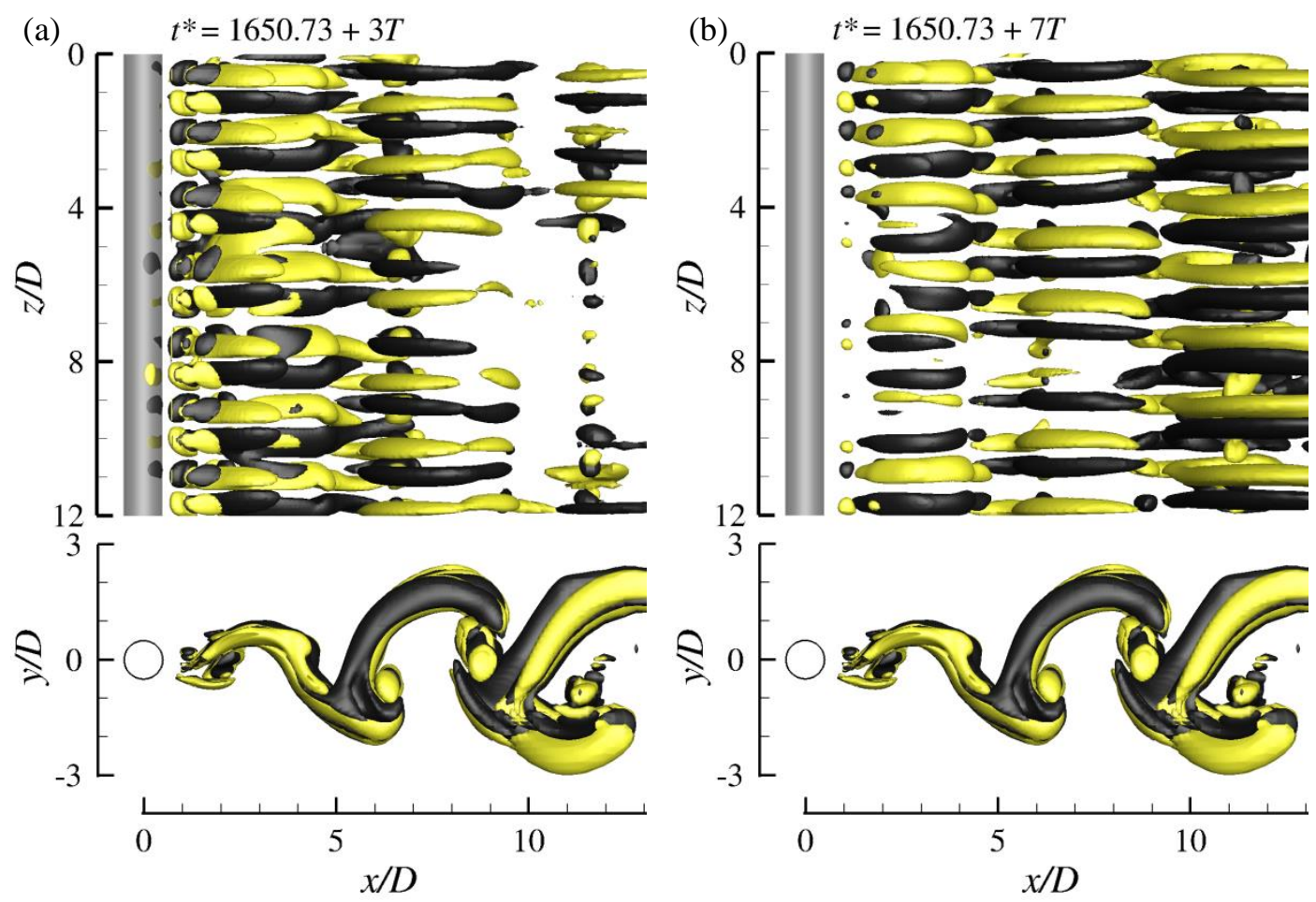

Fig. 11. Mode $\mathrm{C}$ structures at $R e=240$ captured by the iso-surfaces of $\omega_{x}= \pm 0.4$. The Mode $\mathrm{C}$ structures are shown in panels (a) and (b) at $4 T$ apart.

For $R e \geq 240$, the number of Mode $\mathrm{C}$ streamwise vortex pairs along the cylinder span length is no longer fixed over time. For example, this number varies between 7 and 8 for the fully developed flow at $R e=240$, in contrast to a fixed number of 7 at $R e=230$. With further increase in $R e$, more streamwise vortex pairs may be observed along the cylinder span length, and the spanwise wavelength of the increasingly disordered Mode $\mathrm{C}$ structure decreases. This is similar to the decrease in the spanwise wavelength of Mode B in the wake of an isolated circular/square cylinder for $R e=$ 300 to 1000 shown in Jiang and Cheng (2017) when Mode B becomes increasingly disordered with increasing $R e$.

For $R e=240-260$, the non-uniform Mode C structures still appear in pairs along the entire cylinder span length. At $R e=270$, the pairing of the streamwise vortices can sometimes be less obvious. With the increase in $R e$ from 270 to 400 (the highest $R e$ considered in this study), the Mode C structures become increasingly disordered, and the pairing of the streamwise vortices becomes increasingly difficult to be identified. 


\subsubsection{Summary on the wake transition to chaos}

In summary, the wake transition to chaos through the Mode $\mathrm{C}$ flow is as follows. For $R e \leq 230$ where the Mode $\mathrm{C}$ flow structure retains periodicity, the wake is expected to be fully laminar. The wake transition to chaos through the Mode $\mathrm{C}$ flow originates from the uneven growth in strength and the consequent non-uniformity of the Mode $\mathrm{C}$ streamwise vortices along the spanwise direction. With the increase in the non-uniformity of Mode $\mathrm{C}$ from $R e=220$ (Fig. 9) to $R e=230$ (Fig. 10), the flow can no longer stay $2 T$-periodic and undergoes a further period doubling (i.e. period quadrupling) to $4 T$-periodic. The period quadrupling phenomenon suggests that the flow becomes increasingly difficult to preserve periodicity after the Mode $\mathrm{C}$ structure becomes increasingly non-uniform along the spanwise direction. In general, period doubling/quadrupling is a common phenomenon when a dynamic system with periodicity (not only limited to fluid mechanics) is about to transition to chaos (e.g. Pourazarm et al., 2015; Weymouth, 2014; Moskalik and Buchler, 1990). However, such a classical period doubling route to chaos is not observed for the wake transition to chaos through the Mode B flow for flow past an isolated circular/square cylinder, since the Mode B structure is neither uniform along the spanwise direction nor periodic over time (see e.g. the DNS results of Behara and Mittal (2010) and Jiang et al. (2016)). The non-uniformity and non-periodicity of the Mode B structure is possibly a result of the interaction between Modes A and B, since for flow past an isolated circular/square cylinder there are two co-existing 3D wake instability modes, as predicted by Barkley and Henderson (1996) through Floquet stability analysis.

For $R e \geq 240$, the increasingly non-uniform Mode C flow loses periodicity and becomes increasingly disordered with increasing $R e$, such that the wake becomes increasingly chaotic. This process is similar to the wake transition to chaos through the Mode B flow, with the exception that the disordered Mode C flow still possesses the unique feature of Mode $\mathrm{C}$, i.e. the streamwise vortices at respective spanwise locations change signs after each shedding period. 
As suggested by Bloor (1964), the phenomenon of wake transition to turbulence/chaos is closely related to the development of three-dimensionality in the wake of the cylinder. For flow past an isolated circular cylinder, the development of three-dimensionality originates from the 3D Mode B flow structure that becomes increasingly disordered with increasing $R e$. Additional 3D DNS performed in the present study (by using the computational mesh employed in Jiang and Cheng (2017), where mesh convergence was checked at $R e=1000$ ) show that by eliminating the Mode B flow structure through computational manipulations (specifically through reducing $L_{z}$ to be smaller than the unstable spanwise wavelengths of Mode B), the transition to turbulence/chaos is suppressed and the wake remains fully laminar up to at least $R e=1000$. At $R e=1000$, the critical $L_{z} / D$ for the suppression of Mode B and turbulence/chaos is between 0.2 and 0.3. For $L_{z} / D \geq 0.3$, disordered Mode B flow structures are observed in the near wake of the cylinder and the flow is chaotic. For $L_{z} / D \leq 0.2$, Mode B is suppressed and the flow is $2 \mathrm{D}$ and laminar (although the computational domain is $3 \mathrm{D})$.

Similarly, for the present scenario of flow past a circular cylinder with a wire disturbance, it is found that the wake transition to turbulence/chaos may be delayed to higher $R e$ values when the Mode $\mathrm{C}$ streamwise vortices are forced uniform and periodic by using an $L_{z}$ comparable to the spanwise wavelength of Mode C. Additional DNS results show that by reducing $L_{z} / D$ from 12 to 3 , the Mode $\mathrm{C}$ flow also loses periodicity at $R e=230-240$. However, with further decrease in $L_{z} / D$ to 1.5 and 1.0, the Mode C flow loses periodicity and becomes chaotic at increased $R e$ of $240-250$ and $350-360$, respectively. These results further support the suggestion that the wake transition to turbulence/chaos originates from the 3D modal structure developed in the near wake.

\subsection{Flow three-dimensionality}

The degree of flow three-dimensionality is quantified by the statistically stationary streamwise enstrophy $\varepsilon_{x}$ in the near-wake region of $x / D=0-10$. Fig. 12 
shows the relationship between $\varepsilon_{x}$ and $R e$ up to $R e=400$. As shown in the inset of Fig. 12, the degree of flow three-dimensionality exhibits a gradual (rather than a sudden) increase at the onset of the Mode $\mathrm{C}$ instability (of $R e_{c r}=166.4$ ). According to Jiang et al. (2018), a gradual increase in the degree of flow three-dimensionality at the onset of the secondary wake instability corresponds to a non-hysteretic wake instability. This is consistent with the conclusion in section 3.1.3 that the present Mode $\mathrm{C}$ instability is non-hysteretic. As $R e$ exceeds $R e_{c r}$, the degree of flow three-dimensionality increases gradually with increasing $\operatorname{Re}$ (Fig. 12). A minor drop in the increase rate is observed at $R e=220-230$, for which the non-uniform Mode C flow is bounded by $2 T$ - and $4 T$-periodic constraints.

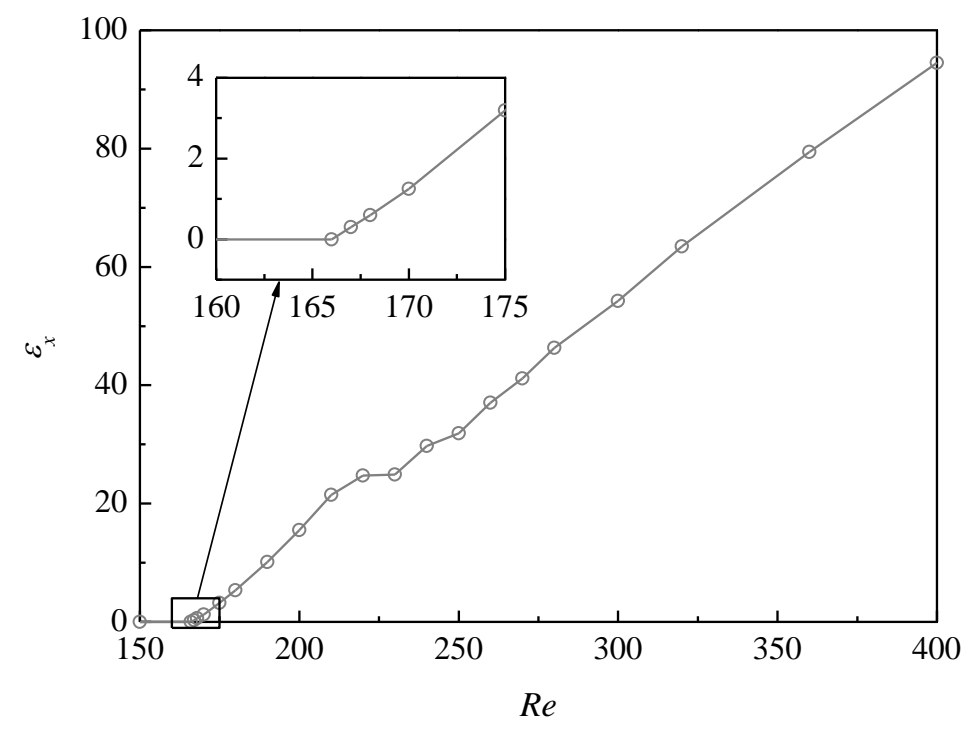

Fig. 12. Variation of the statistically stationary streamwise enstrophy with $R e$.

Fig. 13 shows the primary and streamwise vortex cores captured by the second negative eigenvalue $\lambda_{2}$ of the tensor $\Psi^{2}+\boldsymbol{\Omega}^{2}$, where $\boldsymbol{\Psi}$ and $\boldsymbol{\Omega}$ are the symmetric and antisymmetric parts of the velocity gradient tensor, respectively (Jeong and Hussain, 1995). At $R e=175$, the Mode $C$ streamwise vortices are barely captured by the iso-surfaces of $\lambda_{2}$ (Fig. 13a), since the flow three-dimensionality at $R e$ slightly above $R_{c r}$ is rather weak (Fig. 12). As the degree of flow three-dimensionality increases with increasing $R e$ (e.g. the $\varepsilon x$ value shown in Fig. 12 increases from 3.2 at $R e=175$ 
to 21.5 at $R e=210$ ), the Mode $\mathrm{C}$ streamwise vortices gradually become apparent (e.g. Fig. 13(b) for $R e=210$ ).

Fig. 13 provides 3D DNS evidence that the Mode $\mathrm{C}$ wake instability originates in the braid shear layer region. The primary vortex cores and streamwise vortices are largely independent of one another, with the primary vortex cores being almost 2D. This finding complements previous linear stability analysis results where the spanwise perturbation vorticity field of Mode $\mathrm{C}$ showed instability structures in the braid shear layer region (Sheard et al., 2005a).

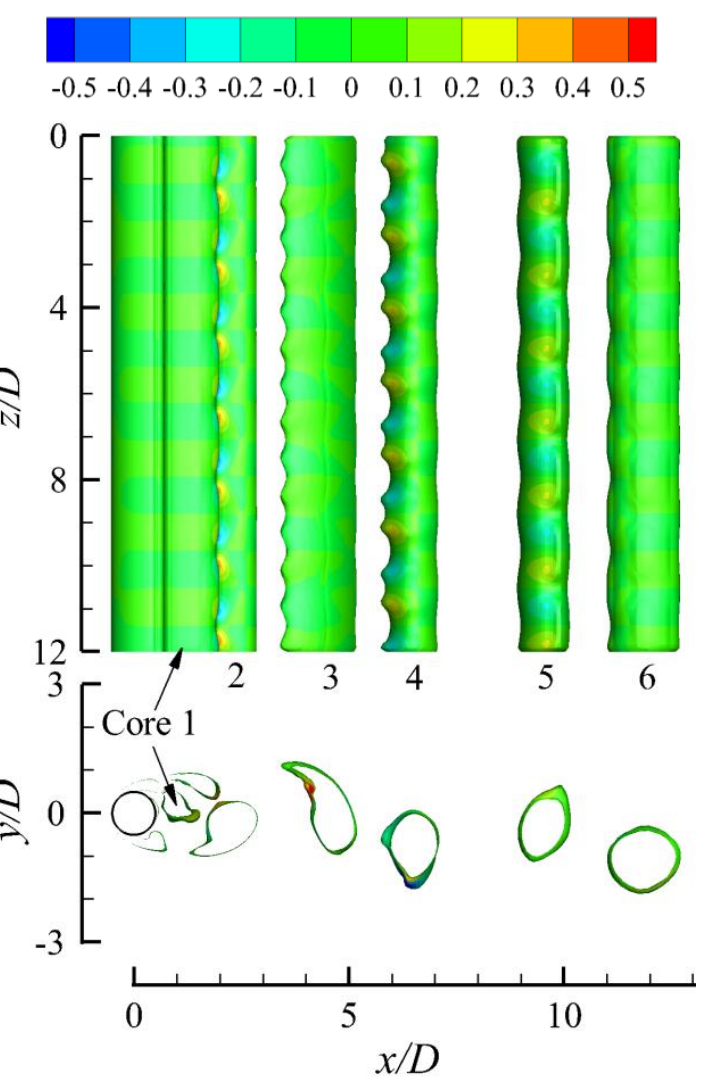

(a)

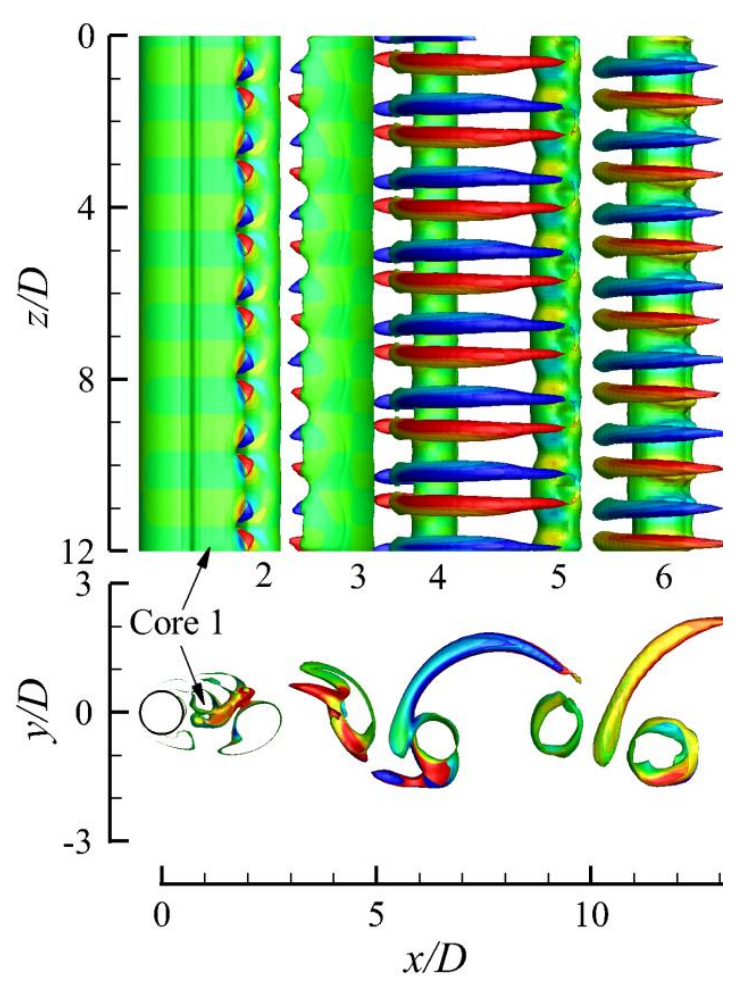

(b)

Fig. 13. Iso-surfaces of $\lambda_{2}=-0.05$ (coloured by $\omega_{x}$ ) of the fully developed flow for (a) $R e=175$, and (b) $R e=210$. The flow is from left to right past the cylinder on the left. The vortex structures are shown at the time instants when the lift coefficient on the main cylinder reaches a local maximum. The primary vortex cores shed from the main cylinder are labelled in each graph. 
Similar to the Mode C wake instability, the Mode B instability, which is responsible for the wake transition to chaos of an isolated circular/square cylinder, also originates in the braid shear layer region (Williamson, 1996; Leweke and Williamson, 1998; Thompson et al., 2001). It is therefore deduced that the transition to chaos in the wake of a bluff body may originate from the instability in the braid shear layer, and the instability in the braid shear layer is induced by a 3D wake instability mode (e.g. Mode B or Mode C).

\subsection{Vortex shedding frequency}

Fig. 14 shows the frequency spectra of $C_{L}$ for the cases with $D_{w} / D=0.05$ and various $R e$ values. The amplitude of $C_{L}$ is normalised with $1 /\left(\sqrt{2} C_{L}^{\prime}\right)$ so that the normalised amplitude becomes 1.0 if the time history of $C_{L}$ is sinusoidal with an arbitrary amplitude. For each case of $R e=R e_{c r}-230$ where the Mode C flow retains strict periodicity, each vortex shedding cycle has an identical time period $T$ (even when the flow is $4 T$-periodic at $R e=230$ ), such that the main frequency peak is represented by a single value $f_{L}(=1 / T)$ with the normalised amplitude equal to 1.0 (Fig. 14). For $R e=R e_{c r}-210$, the time histories of $C_{L}$ are almost $T$-periodic (e.g. Fig. 7a), such that small-amplitude harmonics of $f_{L}$ are observed at $n f_{L}$ ( $n$ being an integer starting from 2). For $R e=220-230$, the time histories of $C_{L}$ become $2 T$-periodic (e.g. Fig. $7 \mathrm{~b}, \mathrm{c})$. Hence in addition to $n f_{L}$, small-amplitude frequency peaks may also appear at $(1 / 2) f_{L}$ and its harmonics $(n / 2) f_{L}$. 


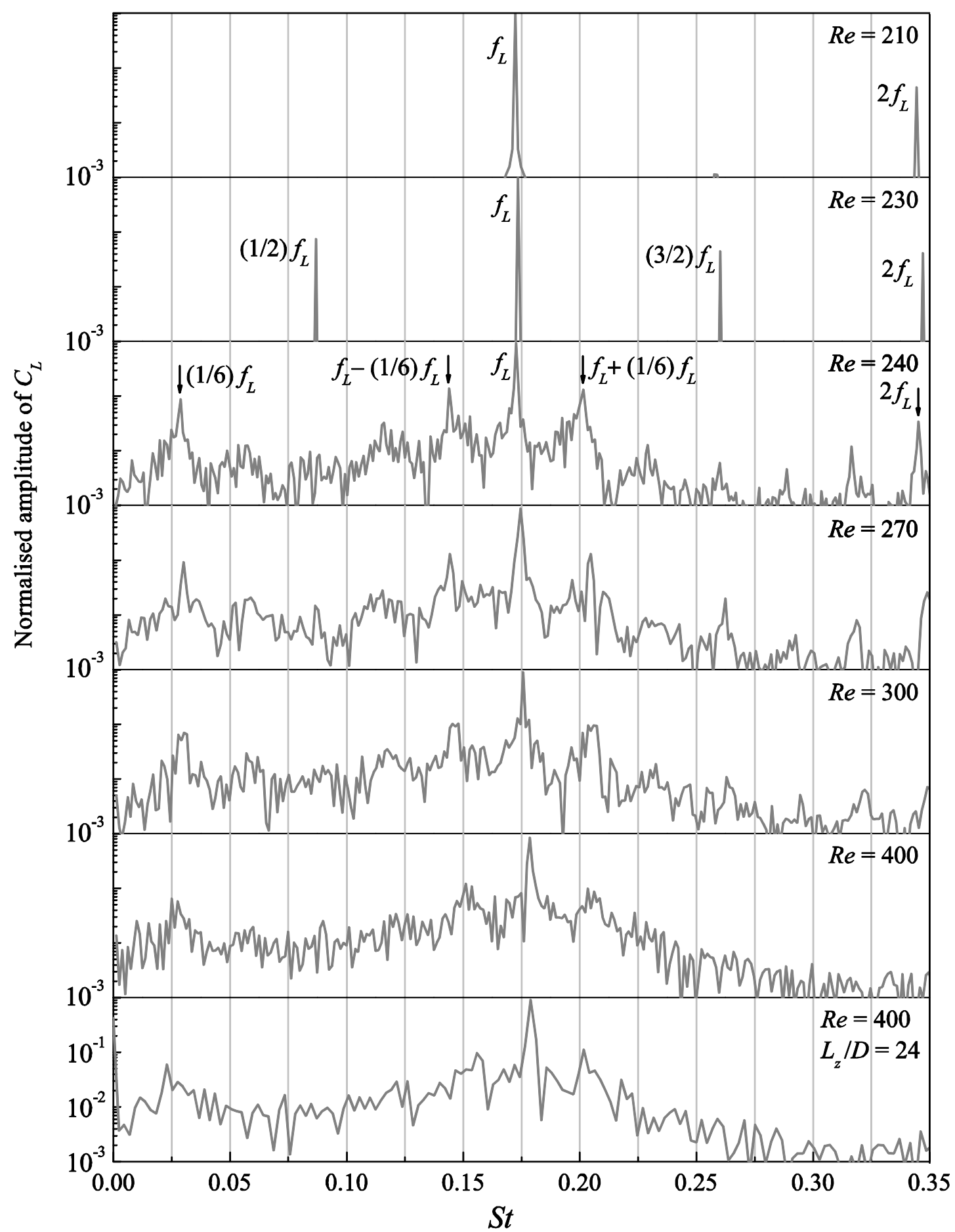

Fig. 14. Frequency spectra of $C_{L}$ for the cases with $D_{w} / D=0.05$ and various $R e$ values.

As the Mode C structure loses periodicity and becomes chaotic for $R e \geq 240$, the frequency spectra become broad-band, with a sharp main peak accompanied by small-scale fluctuations at the two sides of the peak, and the peak amplitude has a 
moderate reduction to $\sim 0.9$. At $R e=240$, a small-amplitude frequency peak is observed at (1/6) $f_{L}$, which is due to the modulation of the time history of $C_{L}$ at a frequency of approximately (1/6) $f_{L}$ (Fig. 7d). The frequency peaks at $f_{L}$ and (1/6) $f_{L}$ also give rise to small-amplitude harmonics at $\left(f_{L}-(1 / 6) f_{L}\right)$ and $\left(f_{L}+(1 / 6) f_{L}\right)$. With the increase in $R e$, similar small-amplitude frequency peaks are also observed at approximately $(1 / 6) f_{L},\left(f_{L}-(1 / 6) f_{L}\right)$ and $\left(f_{L}+(1 / 6) f_{L}\right)$, yet they become less sharp-peaked as the flow becomes increasingly chaotic with increasing $R e$. The bottom two panels of Fig. 14 show the frequency spectra for $R e=400$ with $L_{z} / D=12$ and 24, where similar features are observed, which suggests that the frequency peak at approximately $(1 / 6) f_{L}$ is not influenced by $L z$.

The $S t-R e$ relationships for an isolated circular cylinder and a circular cylinder with a near-wake wire of either $D_{w} / D=0.01$ or $D_{w} / D=0.05$ are shown in Fig. 15 . Based on 2D DNS, it is found that $S t$ decreases gradually with increase in $D_{w} / D$, which is due to the decrease in the flow rate around the cylinder (due to the placement of wire) and consequently the increase in the shear layer thickness. The critical $R e$ for the onset of vortex shedding, i.e. the left tip of the $S t-R e$ relationship shown in Fig. 15, increases from 47 for an isolated cylinder to 52 for $D_{w} / D=0.01$ and 55 for $D_{w} / D=$ 0.05. The suppression of vortex shedding by the placement of a near-wake wire is because the shear layer on the wire side of the main cylinder is weakened by the wire (Strykowski and Sreenivasan, 1990), and also because the flow rate around the cylinder is reduced. 


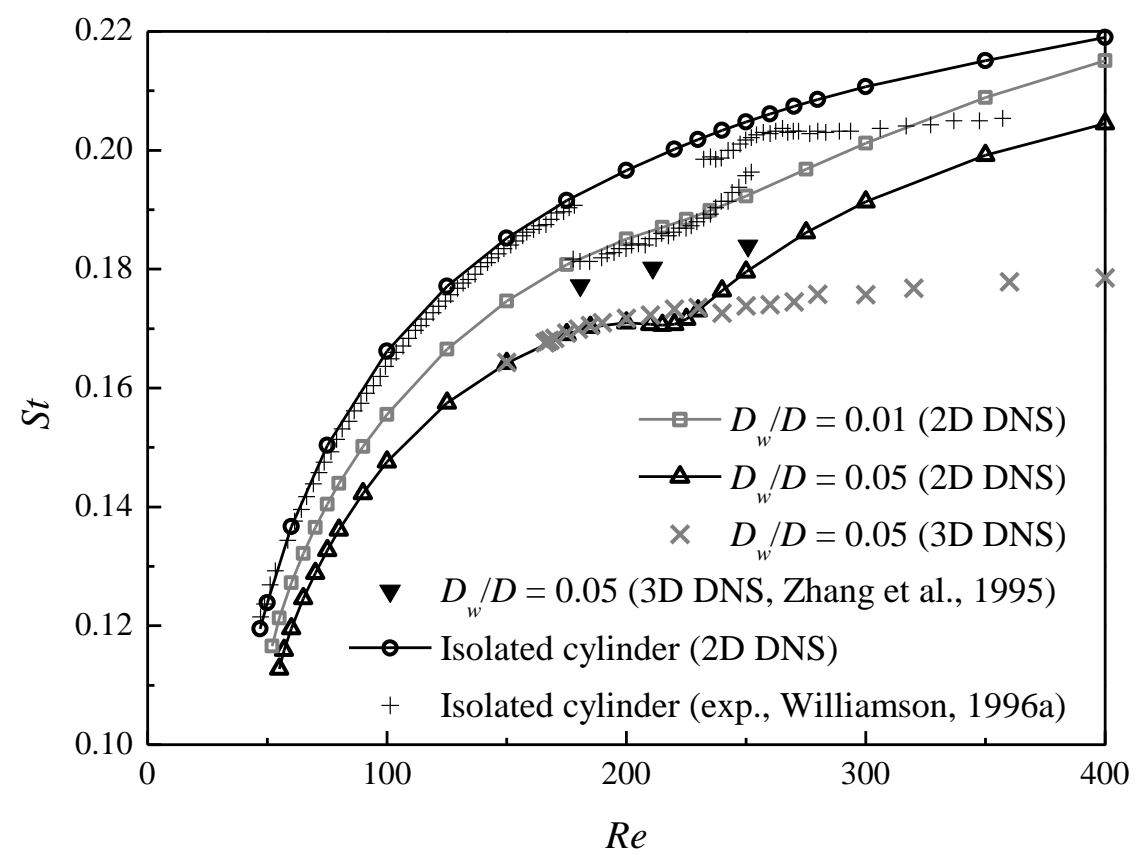

Fig. 15. The $S t-R e$ relationships over the laminar and 3D wake transition regimes.

For the 3D flows, $S t$ is determined based on the peak frequency identified in the frequency spectra of $C_{L}$. At the onset of the Mode $C$ instability of $R e_{c r}=166.4$, a gradual (rather than a sudden) deviation of the 3D St values from their 2D counterparts is observed (Fig. 15), since here the Mode C instability is supercritical. Similarly, the 3D hydrodynamic forces on the main cylinder, such as the time-averaged drag coefficient and root-mean-square lift coefficient, also deviate gradually from their $2 \mathrm{D}$ counterparts as $R e$ exceeds $R e_{c r}$ (graphs omitted for simplicity) due to the supercritical nature of the Mode $\mathrm{C}$ instability. In contrast, since the Mode A instability for an isolated circular/square cylinder is subcritical (Williamson, 1996; Jiang et al., 2018), a sudden drop of $S t$ is observed when the flow transitions from 2D to Mode A (see, e.g., Fig. 15).

Zhang et al. (1995) and Yildirim et al. (2013a) both reported that the vortex shedding frequency of the Mode $\mathrm{C}$ flow is lower than that of the Mode A flow (see e.g. Fig. 15). Here with the further aid of the corresponding 2D DNS results shown in Fig. 15 , it is found that the difference in the vortex shedding frequency for the Modes A and $\mathrm{C}$ flows is actually to a great extent affected by the geometric configurations in 
the $x-y$ plane (i.e. with or without a wire), in addition to the presence of the 3D modes. Compared with the 2D vortex shedding frequency for an isolated cylinder, the placement of a near-wake wire of $D_{w} / D=0.01$ would result in an obvious reduction in the $2 \mathrm{D}$ shedding frequency already. The reduction in the $2 \mathrm{D}$ shedding frequency is more pronounced when $D_{w} / D$ is increased (e.g. from 0.01 to 0.05 ).

\section{Conclusions}

This paper presents 3D DNS of flow past a circular cylinder with a near-wake wire disturbance over a range of $R e$ up to 400 . Additional Floquet stability analysis shows that the wake is unstable to Mode $\mathrm{C}$ only, without potential interactions with other wake modes, which raises this setup as a good candidate for the investigation of the evolution of the Mode $\mathrm{C}$ flow with $R e$. The predicted critical $R e$ and the corresponding spanwise wavelength at the onset of Mode $\mathrm{C}$ are $\left(\operatorname{Re}_{c r}, \lambda_{c r} / D\right)=(166.4$, $1.5)$ for $D_{w} / D=0.05$ and $(169.1,1.3)$ for $D_{w} / D=0.01$. Here the Mode C instability is supercritical, such that there are no sudden changes in the Strouhal number, hydrodynamic forces or the degree of flow three-dimensionality at the onset of Mode C.

The evolutions of the Mode $\mathrm{C}$ wake structure with time and $R e$ are examined and discussed based on the results obtained with $D_{w} / D=0.05$. With the increase in $R e$, the fully developed Mode C flow undergoes an evolution sequence of: (i) uniform periodic regime for $R e=R e_{c r}-210$, (ii) non-uniform periodic regime for $R e=220-$ 230 , and (iii) chaotic regime for $R e \geq 240$. In the uniform periodic regime ( $R e=R e_{c r}-$ 210), the fully developed Mode C structure is highly repeatable along the spanwise direction. The flow structure is $2 T$-periodic but retains a spatiotemporal symmetry $\omega_{x}(x, y, z, t)=\omega_{x}(x, y, z \pm \lambda / 2, t+T)$ every $1 T$. In the non-uniform periodic regime $(R e=220-230)$, the uniform Mode C structure only persists for a short period of time before it evolves spontaneously into a more stable pattern of non-uniform Mode C. The slightly non-uniform Mode $\mathrm{C}$ structure remains $2 T$-periodic at $R e=220$ but 
undergoes a period quadrupling to $4 T$-periodic at $R e=230$ as the non-uniformity increases with increasing $R e$. In the chaotic regime ( $R e \geq 240)$, the Mode C flow loses periodicity and becomes increasingly disordered with increasing $R e$.

Based on the scenarios of flow past an isolated circular/square cylinder (e.g. Williamson, 1996; Jiang et al., 2018) and the present scenario of flow past a circular cylinder with a near-wake wire disturbance, it is deduced that the transition to chaos in the wake of a bluff body may originate from the instability in the braid shear layer region, and the instability in the braid shear layer region is induced by a $3 \mathrm{D}$ wake instability mode (e.g. Mode B or Mode C).

For the present scenario, the wake transition to chaos through the Mode $\mathrm{C}$ flow originates from the uneven growth in strength and the consequent non-uniformity of the Mode $\mathrm{C}$ streamwise vortices along the spanwise direction. The period quadrupling phenomenon at $R e=230$ suggests that the Mode $\mathrm{C}$ flow becomes increasingly difficult to preserve periodicity after the Mode $\mathrm{C}$ structure becomes increasingly non-uniform along the spanwise direction. For $R e \geq 240$, the increasingly non-uniform Mode $\mathrm{C}$ flow loses periodicity and becomes increasingly disordered with increasing $R e$, such that the wake becomes increasingly chaotic. The increasingly disordered Mode C and Mode B flows share some similarities: (i) the instability of both modes originates from the braid shear layer region but not the primary vortex cores, (ii) the streamwise vortices of both modes appear in pairs, (iii) with increasing $R e$ the spanwise wavelength of the mode (hence the spatial scale of turbulence) decreases.

\section{Acknowledgements}

The authors would like to acknowledge the support from the National Key R\&D Program of China (Project ID: 2016YFE0200100). The first author would like to acknowledge the support from the Australian Research Council through the DECRA scheme (DE190100870) and the support from the Australian Government and The University of Western Australia by providing IPRS and APA scholarships for a 
doctoral degree (for an early draft of the manuscript in the PhD thesis - Jiang (2017)). This work was supported by resources provided by the Pawsey Supercomputing Centre with funding from the Australian Government and the Government of Western Australia.

\section{References}

Akbar, T., Bouchet, G., Dušek, J., 2011. Numerical investigation of the subcritical effects at the onset of three-dimensionality in the circular cylinder wake. Physics of Fluids 23, 094103.

Barkley, D., Henderson, R.D., 1996. Three-dimensional Floquet stability analysis of the wake of a circular cylinder. Journal of Fluid Mechanics 322, 215-241.

Barkley, D., Tuckerman, L.S., Golubitsky, M., 2000. Bifurcation theory for three-dimensional flow in the wake of a circular cylinder. Physical Review E 61, 5247-5252.

Behara, S., Mittal, S., 2010. Wake transition in flow past a circular cylinder. Physics of Fluids 22, 114104.

Blackburn, H.M., Sheard, G.J., 2010. On quasiperiodic and subharmonic Floquet wake instabilities. Physics of Fluids 22, 031701.

Bloor, M.S., 1964. The transition to turbulence in the wake of a circular cylinder. Journal of Fluid Mechanics 19, 290-304.

Braza, M., Faghani, D., Persillon, H., 2001. Successive stages and the role of natural vortex dislocations in three-dimensional wake transition. Journal of Fluid Mechanics 439, 1-41.

Cantwell, C.D., et al., 2015. Nektar++: An open-source spectral/hp element framework. Computer Physics Communications 192, 205-219.

Carmo, B.S., Sherwin, S.J., Bearman, P.W., Willden, R.H.J., 2008. Wake transition in the flow around two circular cylinders in staggered arrangements. Journal of Fluid Mechanics 597, $1-29$.

Dušek, J., Le Gal, P., Fraunié, P., 1994. A numerical and theoretical study of the first Hopf bifurcation in a cylinder wake. Journal of Fluid Mechanics 264, 59-80.

Henderson, R.D., 1997. Nonlinear dynamics and pattern formation in turbulent wake transition. Journal of Fluid Mechanics 352, 65-112. 
Henderson, R.D., Barkley, D., 1996. Secondary instability in the wake of a circular cylinder. Physics of Fluids 8, 1683-1685.

Issa, R.I., 1986. Solution of implicitly discretized fluid flow equations by operator-splitting. Journal of Computational Physics 62, 40-65.

Jiang, H., Cheng, L., Draper, S., An, H., Tong, F., 2016. Three-dimensional direct numerical simulation of wake transitions of a circular cylinder. Journal of Fluid Mechanics 801, $353-391$.

Jiang, H., 2017. Wake transition of a circular cylinder. Doctor of Philosophy, The University of Western Australia.

Jiang, H., Cheng, L., 2017. Strouhal-Reynolds number relationship for flow past a circular cylinder. Journal of Fluid Mechanics 832, 170-188.

Jiang, H., Cheng, L., Draper, S., An, H., 2017a. Two- and three-dimensional instabilities in the wake of a circular cylinder near a moving wall. Journal of Fluid Mechanics 812, 435-462.

Jiang, H., Cheng, L., An, H., 2017b. On numerical aspects of simulating flow past a circular cylinder. International Journal for Numerical Methods in Fluids, 85, 113-132.

Jiang, H., Cheng, L., Draper, S., An, H., 2017c. Prediction of the secondary wake instability of a circular cylinder with direct numerical simulation. Computers \& Fluids 149, 172-180.

Jiang, H., Cheng, L., 2018. Hydrodynamic characteristics of flow past a square cylinder at moderate Reynolds numbers. Physics of Fluids 30, 104107.

Jiang, H., Cheng, L., An, H., 2018. Three-dimensional wake transition of a square cylinder. Journal of Fluid Mechanics 842, 102-127.

Karniadakis, G.E., Triantafyllou, G.S., 1992. Three-dimensional dynamics and transition to turbulence in the wake of bluff objects. Journal of Fluid Mechanics 238, 1-30.

Landau, L. D., Lifshitz, E. M., 1976. Mechanics, 3rd Edn. Pergamon.

Lei, C., Cheng, L., Kavanagh, K., 2001. Spanwise length effects on three-dimensional modelling of flow over a circular cylinder. Computer Methods in Applied Mechanics and Engineering 190, 2909-2923.

Leweke, T., Williamson, C.H.K., 1998. Three-dimensional instabilities in wake transition. European Journal of Mechanics - B/Fluids 17, 571-586. 
Moskalik, P., Buchler, J.R., 1990. Resonances and period doubling in the pulsations of stellar models. Astrophysical Journal 355, 590-601.

Ng, Z.Y., Vo, T., Sheard, G.J., 2018. Stability of the wakes of cylinders with triangular cross-sections. Journal of Fluid Mechanics 844, 721-745.

Park, D., Yang, K.S., 2018. Effects of planar shear on the three-dimensional instability in flow past a circular cylinder. Physics of Fluids 30, 034103.

Posdziech, O., Grundmann, R., 2001. Numerical simulation of the flow around an infinitely long circular cylinder in the transition regime. Theoretical and Computational Fluid Dynamics 15, 121-141.

Pourazarm, P., Lackner, M., Modarres-Sadeghi, Y., 2015. Period-doubling route to chaos in a two-degree-of-freedom flexibly-mounted rigid plate placed in water. Journal of Fluids and Structures 57, 375-390.

Rao, A., Leontini, J., Thompson, M.C., Hourigan, K., 2013. Three-dimensionality in the wake of a rotating cylinder in a uniform flow. Journal of Fluid Mechanics 717, 1-29.

Rao, A., Leontini, J.S., Thompson, M.C., Hourigan, K., 2017. Three-dimensionality of elliptical cylinder wakes at low angles of incidence. Journal of Fluid Mechanics 825, 245-283.

Sheard, G.J., Thompson, M.C., Hourigan, K., 2003. From spheres to circular cylinders: the stability and flow structures of bluff ring wakes. Journal of Fluid Mechanics 492, 147-180.

Sheard, G.J., Thompson, M.C., Hourigan, K., 2004. From spheres to circular cylinders: non-axisymmetric transitions in the flow past rings. Journal of Fluid Mechanics 506, 45-78.

Sheard, G.J., Thompson, M.C., Hourigan, K., 2005a. Subharmonic mechanism of the mode C instability. Physics of Fluids 17, 111702.

Sheard, G.J., Thompson, M.C., Hourigan, K., Leweke, T., 2005b. The evolution of a subharmonic mode in a vortex street. Journal of Fluid Mechanics 534, 23-38.

Sheard, G.J., Fitzgerald, M.J., Ryan, K., 2009. Cylinders with square cross-section: wake instabilities with incidence angle variation. Journal of Fluid Mechanics 630, 43-69.

Strykowski, P.J., Sreenivasan, K.R., 1990. On the formation and suppression of vortex 'shedding' at low Reynolds numbers. Journal of Fluid Mechanics 218, 71-107.

Thompson, M.C., Leweke, T., Williamson, C.H.K., 2001. The physical mechanism of transition in 
bluff body wakes. Journal of Fluids and Structures 15, 607-616.

Weymouth, G.D., 2014. Chaotic rotation of a towed elliptical cylinder. Journal of Fluid Mechanics 743, 385-398.

Williamson, C.H.K., 1996. Three-dimensional wake transition. Journal of Fluid Mechanics 328, 345-407.

Yildirim, I., Rindt, C.C.M., van Steenhoven, A.A., 2013a. Mode C flow transition behind a circular cylinder with a near-wake wire disturbance. Journal of Fluid Mechanics 727, 30-55.

Yildirim, I., Rindt, C.C.M., van Steenhoven, A.A., 2013b. Energy contents and vortex dynamics in Mode-C transition of wired-cylinder wake. Physics of Fluids 25, 054103.

Zhang, H.Q., Fey, U., Noack, B.R., König, M., Eckelmann, H., 1995. On the transition of the cylinder wake. Physics of Fluids 7, 779-794. 